\title{
Strombomonas Deflandre (Euglenophyceae) em ambientes aquáticos na Área de Proteção Ambiental do Ibirapuitã, Estado do Rio Grande do Sul, Brasil
}

\author{
Sandra Maria Alves-da-Silva ${ }^{1,2}$, Isabele Corino Klein ${ }^{1}$ e Isadora Lieske ${ }^{1}$ \\ Recebido: 24.02.2017; aceito: 31.07.2017
}

\begin{abstract}
Strombomonas Deflandre (Euglenophyceae) in aquatic environments in the Área de Proteção Ambiental de Ibirapuitã, Rio Grande do Sul State, Brazil). This paper is the result of the taxonomic study of the genus Strombomonas Defl. in the Área de Proteção Ambiental do Ibirapuitã located in the southwest of Rio Grande do Sul State, Brazil. Samples were collected between March 2011 and March 2012 from ten sites, six in the Ibirapuitã River and four in lotic and lentic environments near the river. A qualitative analysis of 26 samples resulted in the identification of 18 specific and infraspecific taxa. A new combination is proposed: Strombomonas scabra (Playf.) S.M. Alves-da-Silva comb. nov. Strombomonas brasiliensis Conf. was registered for the first time in the State. Strombomonas verrucosa (Daday) Defl. var. verrucosa was the most frequent, occurring in a broad spectrum of environmental variables, such as electrical conductivity, $\mathrm{pH}$, water temperature, silica and organic matter.
\end{abstract}

Keywords: Euglenophyta, Pampa biome, southwest of Rio Grande do Sul State, taxonomy

RESUMO - (Strombomonas Deflandre (Euglenophyceae) em ambientes aquáticos na Área de Proteção Ambiental do Ibirapuitã, Estado do Rio Grande do Sul, Brasil). Este trabalho é resultado do estudo taxonômico de táxons do gênero Strombomonas Defl. na Área de Proteção Ambiental do Ibirapuitã, localizada no sudoeste do Estado do Rio Grande do Sul. As amostras foram coletadas entre março/2011 e março/2012 abrangendo 10 locais, sendo seis no rio Ibirapuitã e quatro em ambientes lóticos e lênticos próximos a esse rio. A análise qualitativa de 26 amostras resultou na identificação de 18 táxons específicos e infraespecíficos. É proposta uma nova combinação Strombomonas scabra (Playf.) S.M. Alves-da-Silva comb. nov. Strombomonas brasiliensis Conf. é o primeiro registro para o Estado. Strombomonas verrucosa (Daday) Defl. var. verrucosa foi muito frequente ocorrendo em amplo espectro de variáveis ambientais como: condutividade elétrica, $\mathrm{pH}$, temperatura da água, sílica e matéria orgânica.

Palavras-chave: bioma Pampa, Euglenophyta, sudoeste do RS, taxonomia

\section{Introdução}

Nas últimas décadas a taxonomia das Euglenophyta (Euglenozoa, Euglenida) tem passado por grandes mudanças taxonômicas com o advento de estudos moleculares.

As Euglenophyceae (Euglenoidea fototróficas) são uma linhagem importante dentro de Euglenida, Euglenozoa. A maioria das cerca de 3.000 espécies descritas são de vida livre, flagelados unicelulares, fototróficas, com um a vários plastídios de origem secundária, três membranas delimitadoras e clorofilas $a$ e $b$; mas, a linhagem inclui também espécies incolores que perderam a sua capacidade de fotossíntese (Bicudo \& Menezes 2016).

O presente trabalho é a continuação do estudo taxonômico de Euglenophyta desenvolvido na Área de Proteção Ambiental (APA) do Rio Ibirapuitã, dentro do programa de Pesquisas Ecológicas de Longa Duração (PELD- CNPq), sítio 25- Campos

1. Fundação Zoobotânica do Rio Grande do Sul, Museu de Ciências Naturais, Seção de Botânica de Criptógamas, Rua Dr. Salvador França, 1427, 90690-000 Porto Alegre, RS, Brasil

2. Autor para correspondência: sandra-silva@fzb.rs.gov.br 
Sulinos. A APA é uma Unidade de Conservação Federal (UC) do Bioma Pampa, com uma extensão de 316.882,75 hectares, localizada no sudoeste do Rio Grande do Sul (Silva 2010), junto à fronteira Brasil e Uruguai, entre as coordenadas $29^{\circ} 59^{\prime} 08^{\prime \prime} \mathrm{S}$

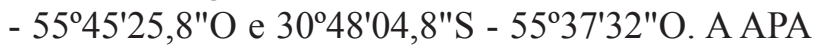
abriga parte da bacia Hidrográfica do Rio Ibirapuitã, sendo amostrados ambientes lóticos e lênticos no rio e ambientes próximos, resultando no registro de uma flora rica em Euglenophyceae.

Quatro gêneros já foram publicados: Cryptoglena Marin \& Melkonian emend. Kosmala \& Zakrýs, Phacus Dujardin e Monomorphina (Ehrenberg) Mereschkowsky emend. Kosmala \& Zakrýs (Alvesda-Silva \& Klein 2015) e o gênero Trachelomonas Ehrenberg emend. Deflandre (Alves-da-Silva et al. 2016). Este será o terceiro trabalho abrangendo os táxons do gênero Strombomonas. Este gênero, assim como as Trachelomonas, se caracteriza por apresentar uma carapaça envolvendo uma célula metabólica, semelhante as Euglena, chamada de lorica. A morfologia da lorica é uma característica amplamente utilizada para a identificação destes dois gêneros (Deflandre 1930, Huber-Pestalozi 1955, Popova 1966, Leedale 1967, Starmach 1983, Tell \& Conforti 1986).

Desde que o gênero foi proposto por Deflandre (1930) sua validade tem sido discutida (Alves-daSilva et al. 2013a). Marin et al. (2003) realizaram estudos com biologia molecular e propuseram que os táxons destes dois gêneros loricados deveriam ser incorporados ao gênero Trachelomonas. No entanto, este e outros estudos recentes com multigenes e um maior número de representantes indicou que os gêneros Strombomonas e Trachelomonas devem continuar separados com base em filogenia molecular, na ontogenia do desenvolvimento da lorica e padrão de redução das estrias da película (Brosnan et al. 2005, Triemer et al. 2006, Ciugulea et al. 2008, Kim \& Shin 2008, Kim et al. 2010, Linton et al. 2010 e Kim et al. 2015).

O impacto da filogenia moderna sobre taxonomia de euglenoides em especial ao nível molecular têm acarretado mudanças taxonômicas, o que leva ao número de espécies aceitas estarem em constante transição. As Strombomonas para Triemer \& Zakrýs (2015) possuem 74 espécies e infraespécies, enquanto Guiry \& Guiry (2015) citam 85 espécies e infraespécies taxonomicamente aceitas.

Os indivíduos de Strombomonas são microscópicos, pigmentados, solitários, livre-natantes graças a dois flagelos, dos quais somente um emerge do reservatório, a coloração da lorica varia de incolor, amarelada até castanha escura conforme a espécie, podendo haver impregnação de sais do meio, predominantemente de sílica e ferro. A parede da lorica pode ser lisa, mas a grande maioria apresenta aglutinações do meio com aspecto granuloso ou rugoso. O polo anterior tem um espessamento anelar, ou um colarinho de forma e dimensões variadas, com terminação indistinta, e o polo posterior pode ou não se atenuar em um processo caudal cônico, de diferente dimensão. O citoplasma apresenta numerosos cloroplastos discoides ou elípticos, e em algumas espécies são acompanhados de haplopirenoides ou duplopirenoides. Grãos de paramido são elípticos de mesma dimensão. A vista apical circular, elíptica, triangular ou angulosa é outra característica complementar importante em alguns táxons, pois permite distinguir espécies e infraespécies.

Os táxons desse gênero apresentam ampla distribuição geográfica (Bourrelly 1970, Bicudo \& Menezes 2005). Quanto ao hábitat ocorrem predominantemente na comunidade fitoplanctônica, mas podem ser encontradas no benton e perifíton, em ambientes dulciaquícolas rasos, lóticos e lênticos como: rios com fluxo lento, margens de ilhas, lagoas, lagos, açudes, reservatórios, banhados, viveiro de peixes e em corpos de água estagnada e efêmeras (Rino 1972, Tell \& Conforti 1986, Philipose 1988, Xavier 1988, Menezes \& Fernandes 1989, Menezes 1992, Alves-da-Silva \& Torres, 1994, Xavier 1994, Alves-da-Silva \& Ávila 1997, Wolowski 1998, Alvesda-Silva \& Crossetti 1999, Alves-da-Silva \& Bicudo 2003, 2006, Wolowski \& Walne 2007, Araújo et al. 2012, Alves-da-Silva et al. 2013a, b, Duangjan \& Wołowski 2013). Alguns autores como Alves-daSilva \& Bicudo (2003), Conforti \& Zalocar (2003), Salazar (2004) e Alves-da-Silva \& Kurrle (2009) citam o gênero em ecossistemas com macrófitas aquáticas e Alves-da-Silva \& Tamanaha (2008) em ambientes utilizados para rizipiscicultura.

A maioria das Strombomonas ocorre em pequeno número de indivíduos por lâmina, dificultando estudos populacionais (Alves-da-Silva et al. 2013a), sendo necessário a observação de um maior número de lâminas por unidade amostral. As euglenofíceas de maneira geral, quando observadas em microscópio óptico entre lâmina e lamínula, se locomovem em direção ao sedimento ou matéria orgânica em decomposição em busca de alimentação e proteção. Os indivíduos de Strombomonas em especial, permanecem no meio do sedimento por longos 
períodos, o que torna sua visualização e obtenção de fotomicrografias muito difícil.

O levantamento dos trabalhos realizados no Rio Grande do Sul revelou que existe o registro de 63 espécies e infraespécies de Strombomonas (Alves-daSilva \& Hahn 2001, Alves-da-Silva \& Bicudo 2003, 2006, Alves-da-Silva \& Bridi 2004, Alves-da-Silva \& Tamahana 2008, Alves-da-Silva \& Kurrle 2009, Alves-da-Silva et al. 2007, 2013a, b, 2015, Domingues \& Torgan 2011), sendo este o maior número de táxons dessas microalgas registradas no Brasil. A provável explicação é a existência de poucos especialistas de Euglenophyta e escassas publicações taxonômicas deste gênero no país.

No sudoeste do Estado e mais especificamente na APA do Ibirapuitã, não existia nenhum registro de espécies e infraespécies de Strombomonas.

O objetivo do estudo foi realizar o levantamento taxonômico e algumas considerações ecológicas de táxons do gênero Strombomonas, em ambientes aquáticos subtropicais lênticos e lóticos na Área de Proteção Ambiental (APA) do Ibirapuitã, para ampliar o conhecimento da diversidade e distribuição geográfica dessas algas no Estado e no Brasil.

\section{Material e métodos}

AAPA do Ibirapuitã é dividida longitudinalmente pelo rio Ibirapuitã que nasce no oeste da cidade de Santana do Livramento, percorre por $100 \mathrm{~km}$ a APA no sentido sul-norte, indo desaguar em Alegrete no Rio Ibicuí, que é afluente da bacia do Rio Uruguai. O Rio Ibirapuitã apresenta nível médio anual de 3,40 m (Silva 2011), mas em época de chuva, alaga grandes áreas e recebe carga orgânica oriunda predominantemente da agricultura (arroz irrigado) e pecuária (bovinos e ovinos) [Silva 2010]. O clima da região é subtropical, temperado quente (Conti \& Furlan 2003).

O estudo foi desenvolvido no rio e em ambientes aquáticos no seu entorno, em dez locais (figura 1), sendo seis estações ao longo do rio (E1-E6), dois afluentes lóticos, o Arroio Sarandizinho (E7) e o Rio Ibirapuitã Chico (E10), e dois ambientes lênticos (Lagoa Marginal Sabrito-E8 e Banhado da Maronna-E9). A lista dos locais de coletas de microalgas, com as respectivas coordenadas geográficas georreferenciadas e os municípios abrangidos no estudo encontram-se em Alves-da-Silva e Klein (2015).

Foram realizadas três coletas para obtenção de amostras que abrangeram as estações de outono (março/2011/2012) e primavera (novembro/ dezembro/2011), totalizando 26 amostras.

As amostras foram coletadas na margem, com rede de plâncton com abertura de malha de $30 \mu \mathrm{m}$, sendo padronizadas entre 30 a 40 passagens na subsuperfície da água. Em campo as amostras foram subdivididas em duas subamostras, sendo uma parte mantida viva em refrigeração e a outra foi conservada com formaldeído a 4\%, sendo posteriormente tombada no Herbário Prof. Dr. Alarich R.H. Schultz (HAS) do Museu de Ciências Naturais da Fundação Zoobotânica do Rio Grande do Sul.

Para a análise qualitativa foram observadas em média 25 lâminas semipermanentes por amostra, em aumento de 400 a 1.000 vezes, em microscópio óptico Leica DMLS. As medidas dos táxons foram efetuadas com ocular micrometrada e as fotomicrografias capturadas posicionando-se a lente da câmara digital Sony, modelo DSC-W370, diretamente na ocular do microscópio. Concomitantemente à coleta do material biológico, foram medidas in loco variáveis ambientais como temperatura da água $\left({ }^{\circ} \mathrm{C}\right)$ e $\mathrm{pH}$ utilizando o potenciômetro modelo DMPH-P,

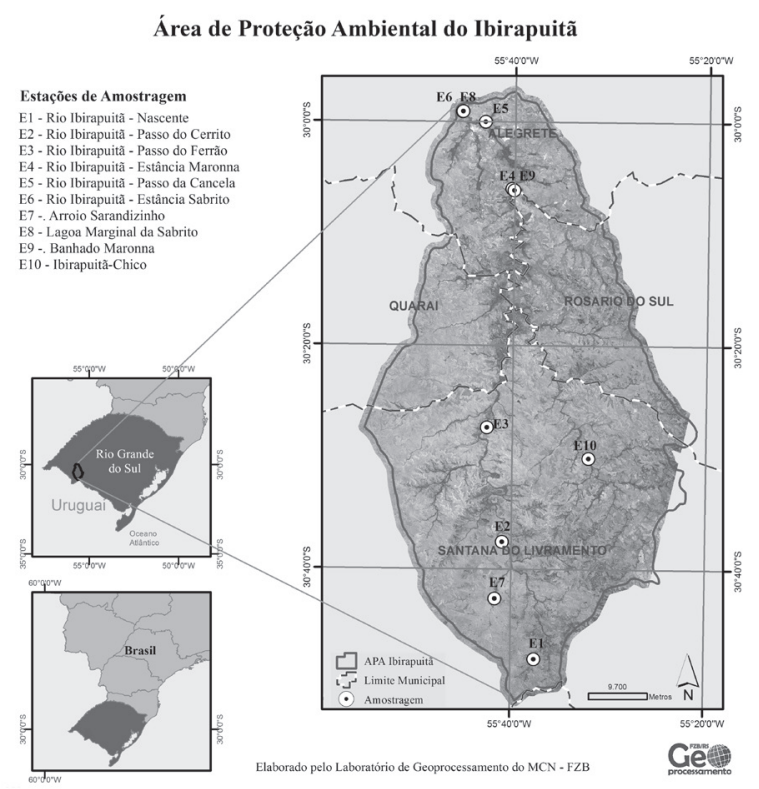

Figura 1. Mapa com a localização das 10 estações amostrais (E1-E10) na APA do Ibirapuitã, Rio Grande do Sul, Brasil. Fonte: Laboratório de Geoprocessamento do Museu de Ciências Naturais da Fundação Zoobotânica do Rio Grande do Sul.

Figure 1. Map showing the location of the 10 sites (E1-E10) in the Área de Proteção Ambiental do Ibirapuitã, Rio Grande do Sul State, Brazil. Source: Geoprocessing Laboratory of the Museum of Natural Sciences of the Zoobotânica Foundation of Rio Grande do Sul. 
condutividade elétrica $\left(\mu \mathrm{S} \mathrm{cm}^{-1}\right)$ com condutivímetro Digimed modelo CD-28 e profundidade máxima (m) com fita métrica. Em laboratório foram medidas matéria orgânica $\left(\mathrm{mg} \mathrm{L}^{-1}\right)$ e sílica $\left(\mathrm{mg} \mathrm{L}^{-1}\right)$ de acordo metodologia do APHA (2005). E a partir da segunda coleta, foi utilizado também, o aparelho Horiba-U50 para medir algumas variáveis.

Foi adotado o sistema de Deflandre (1930), que se baseia fundamentalmente em características da lorica como morfologia, dimensões e ornamentação da parede e vista apical. Foi complementada também às descrições, a relação entre o comprimento e a largura da lorica (Rc/l) de cada indivíduo, estruturas protoplasmáticas como cloroplastos, paramidos e pirenoides. Todas as descrições foram baseadas em observações fenotípicas em microscópio óptico, de espécimes vivos e conservados. São apresentados ainda alguns comentários, a relação do material examinado, distribuição geográfica por região e abreviatura dos Estados do Brasil. A distribuição seguiu Alves-da-Silva \& Menezes (2015), sendo incluída a variedade típica dentro da distribuição da espécie típica. Foram considerados somente os trabalhos que permitem reidentificação dos táxons. Abreviaturas utilizadas nas descrições: comprimento =comp., largura $=$ larg., diâmetro $=$ diâm., cerca $\mathrm{de}=\mathrm{ca}$.

Após a identificação dos táxons foi calculada a frequência de ocorrência dos mesmos, de acordo com a fórmula, onde: $\mathrm{F}=\mathrm{n} .100 / \mathrm{N}$, onde, $\mathrm{n}=$ número de amostras em que uma espécie foi registrada, $\mathrm{N}=$ total de amostras analisadas. As categorias de frequência aplicadas estão de acordo com Matteucci \& Colma (1982), sendo considerado: $>70 \%$ - Muito Frequente (MF), $\leq 70 \%$ e $>40 \%$ - Frequente $(\mathrm{F}), \leq 40 \%$ e $>10 \%$ Pouco Frequente $(\mathrm{PF}), \leq 10 \%$ - Rara (R). A riqueza específica (S) é o número de táxons por amostra.

A identificação em nível de divisão e classe seguiu Hoeck et al. (1995) e para nível específico e infraespecífico dos táxons foram utilizadas obras básicas como: Deflandre (1930), Conrad \& Van Meel (1952), Huber-Pestalozzi (1955), Starmach (1983), Tell \& Conforti (1986), Shi et al. (1999), além de outros trabalhos como: Rino (1972), Bourrelly \& Couté (1978), Yacubson (1980), Xavier (1988, 1994), Menezes \& Fernandes (1989), Cecy (1990), Franceschini (1992), Menezes (1992), Conforti (1993), Alves-da-Silva \& Torres (1994), Jati \& Train (1994), Menezes et al. 1995, Bittencourt-Oliveira (1997), Nogueira \& Leandro-Rodrigues (1999),
Keppeler et al. (2002), Alves-da-Silva\& Bridi (2004), Zuberlin et al. (2004), Delazari-Barroso et. al. 2007, Wolowski \& Walne (2007), Alves-daSilva \& Kurrle (2009), Domingues \& Torgan (2011), Araujo et al. (2012) e Alves-da-Silva et al. (2013a, b), entre outros.

\section{Resultados e Discussão}

Foram registrados táxons do gênero na APA nas 26 unidades amostrais estudadas (tabela 1). Resultando na identificação de 18 táxons de Strombomonas em nível específico e infraespecífico, distribuídos em cinco espécies típicas, sete variedades típicas, quatro variedades não típica e duas formas não típica da espécie.

Levantamento taxonômico

Classe Euglenophyceae

Ordem Euglenales

Família Euglenaceae

Strombomonas Deflandre 1930.

Strombomonas asymmetrica (Roll) Pop., Flora plant. Cryptog. 8(1): 217, pl. 20, fig. 18. 1966. Figuras 2, 3

Lorica elíptica a ligeiramente triangular, 30-33 $\mu \mathrm{m}$ compr., 15-16 $\mu \mathrm{m}$ larg., $\mathrm{Rc} / 1=2$; polo anterior gradativamente atenuado em um colarinho cilíndrico, levemente reto ou oblíquo, bordo crenulado, 4-5 $\mu \mathrm{m}$ alt., $5 \mu \mathrm{m}$ larg.; polo posterior terminando em processo caudal cônico de $5 \mu \mathrm{m}$; parede hialina a amarelada, levemente granulosa; paramidos bastoniformes, ca. $3 \mu \mathrm{m}$ compr.; cloroplastos com duplopirenoides.

Morfologicamente $S$. asymmetrica é próxima de $S$. fluviatilis (Lemm.) Defl. com superposição de medidas entre ambas. A diferença é a presença de um colarinho mais alto e duplopirenoides, e uma Rc/1 menor em $S$. asymmetrica.

Material examinado: BRASIL. Rio GRANDE Do SUL: Alegrete, Estância Sabrito - E6, 29-XI-2011, S.M. Alves-da-Silva (HAS109270).

Distribuição geográfica no Brasil: Norte (AM), Sul (RS).

Strombomonas borystheniensis (Roll) Pop. var. borystheniensis, Flora plant. Cryptog. 8(1): 206, pl. 20, fig. 15. 1966.

Figura 4 


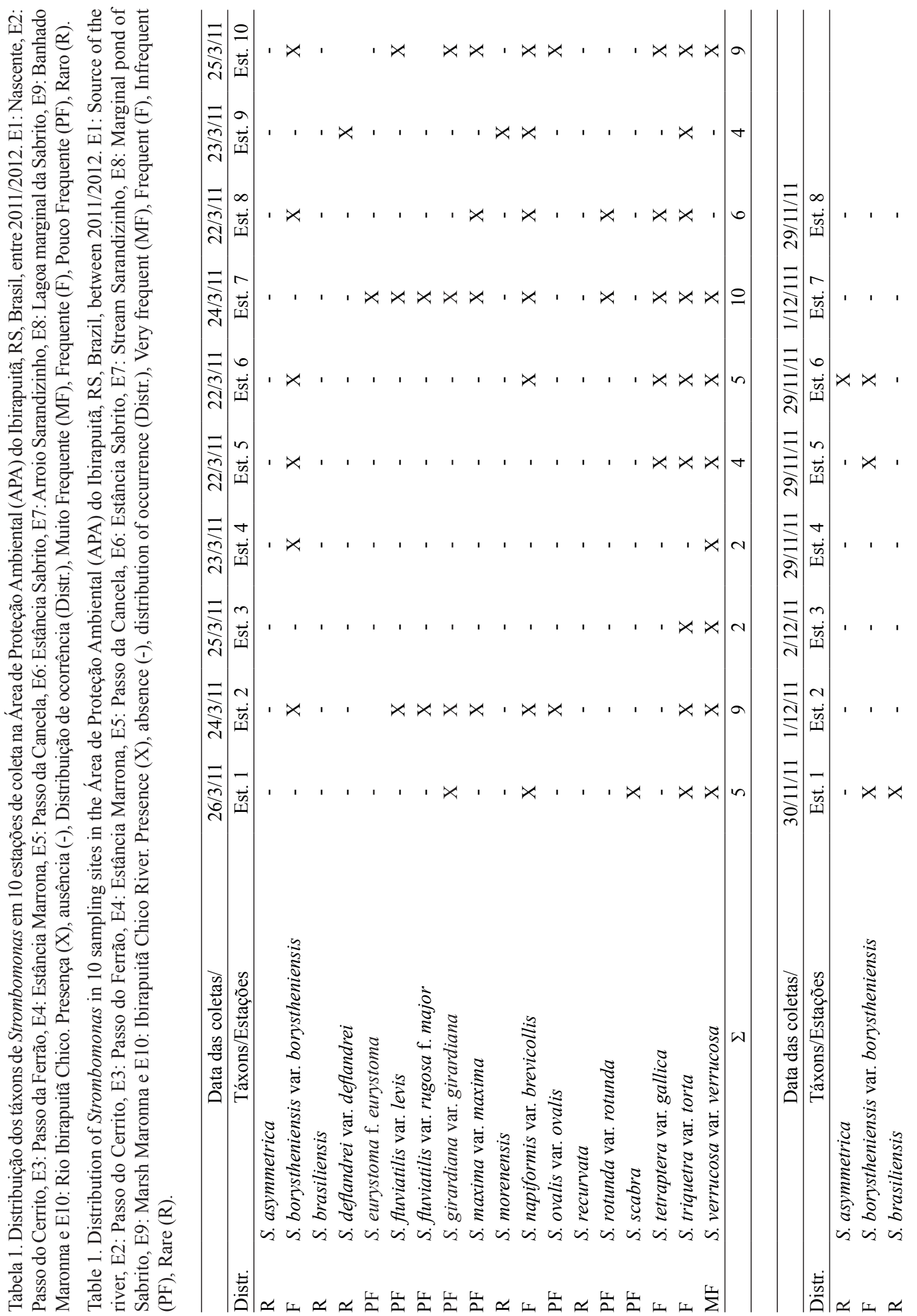




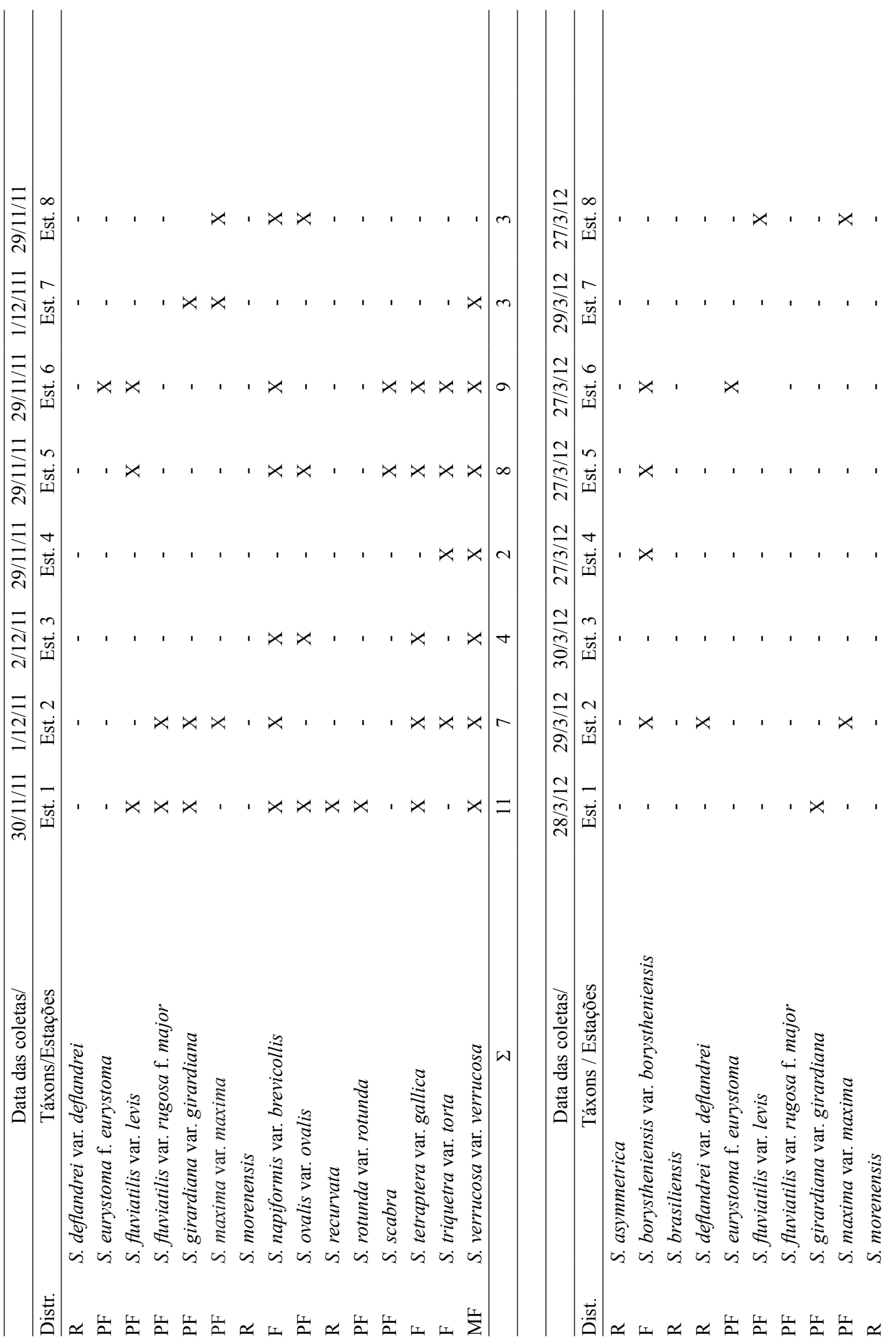




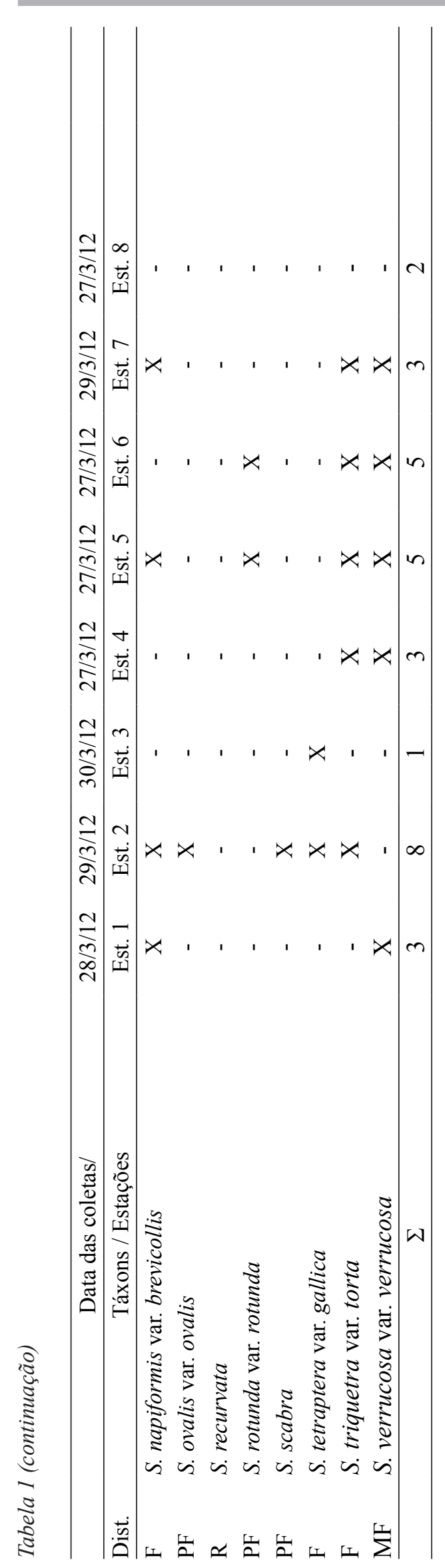

Lorica oblonga ou amplamente elíptica, 25-30,5 $\mu \mathrm{m}$ compr., 17-23 $\mu \mathrm{m}$ larg., Rc/l=1,3-1,5; polo anterior terminando em colarinho baixo, oblíquo, bordo crenulado de 0,8-3,0 $\mu \mathrm{m}$ alt., $6-8 \mu \mathrm{m}$ larg.; polo posterior arredondado; parede verrucosa, espessa, com aglutinações do meio, castanho-claro a média; cloroplastos discoides ou bastoniformes até $5 \mu \mathrm{m}$ diâm.

Esta espécie apresenta semelhança com S. scabra comb. nov. distinguindo-se por apresentar parede mais espessa, colarinho mais baixo e oblíquo.

Material examinado: BRASIL. Rio GRANDE do Sul: Livramento, Passo do Cerrito - E2, 24-III-2011, S.M. Alves-da-Silva (HAS 109251); Alegrete, Estância Maronna - E4, 23-III-2011, S.M. Alves-da-Silva (HAS 109253); Alegrete, Passo da Cancela - E5, 22-III-2011, S.M. Alves-da-Silva (HAS109254); Alegrete, Estância Sabrito - E.6, 22-III-2011, S.M. Alves-da-Silva (HAS 109255); Livramento, Rio Ibirapuitã Chico - E10, 25-III-2011, S.M. Alves-da-Silva (HAS109257); Alegrete, Lagoa Marginal Sabrito - E8, 22-III-2011, S.M. Alves-da-Silva (HAS 109260); Livramento, Nascente - E1, 30-XI-2011, S.M. Alves-da-Silva (HAS109261); Alegrete, Passo da Cancela - E5, 29XI-2011, S.M. Alves-da-Silva (HAS109268); Alegrete, Estância Sabrito - E6, 29-XI-2011, S.M. Alves-daSilva (HAS109270); Livramento, Passo do Cerrito - E2, 29-III-2012, S.M. Alves-da-Silva (HAS109275); Alegrete, Estância Maronna - E4, 27-III-2012, S.M. Alves-da-Silva (HAS109277); Alegrete, Passo da Cancela - E5, 27-III-2012, S.M. Alves-da-Silva (HAS109278); Alegrete, Estância Sabrito - E6, 27-III-2012, S.M. Alves-da-Silva (HAS109279).

Distribuição geográfica no Brasil: Norte (AM), Sul (RS, SC).

Strombomonas brasiliensis Conf., Rev. Hydrobiol. trop. 26 (3): 188-189, pl.III, fig. 7 a,b. 1993.

Figuras 5-7

Lorica fusiforme a oblonga, 36-37 $\mu \mathrm{m}$ (sem espinho) até $41 \mu \mathrm{m}$ compr. (com espinho), 18,5 $\mu \mathrm{m}$ larg.; Rc/l = 1,9-2,2; polo anterior terminando em colarinho cilíndrico, $6 \mu \mathrm{m}$ alt., $4 \mu \mathrm{m}$ larg. ou $8 \mu \mathrm{m}$ larg. com os pequenos espinhos divergentes na região distal; polos levemente acuminados, o posterior truncado à levemente achatado; parede pontuada, castanha-avermelhada clara, com espinhos mais nos polos, espinhos cônicos com extremo final arredondado ou agudo, ca. $2 \mu \mathrm{m}$. compr.; presença de haplopirenoides. 

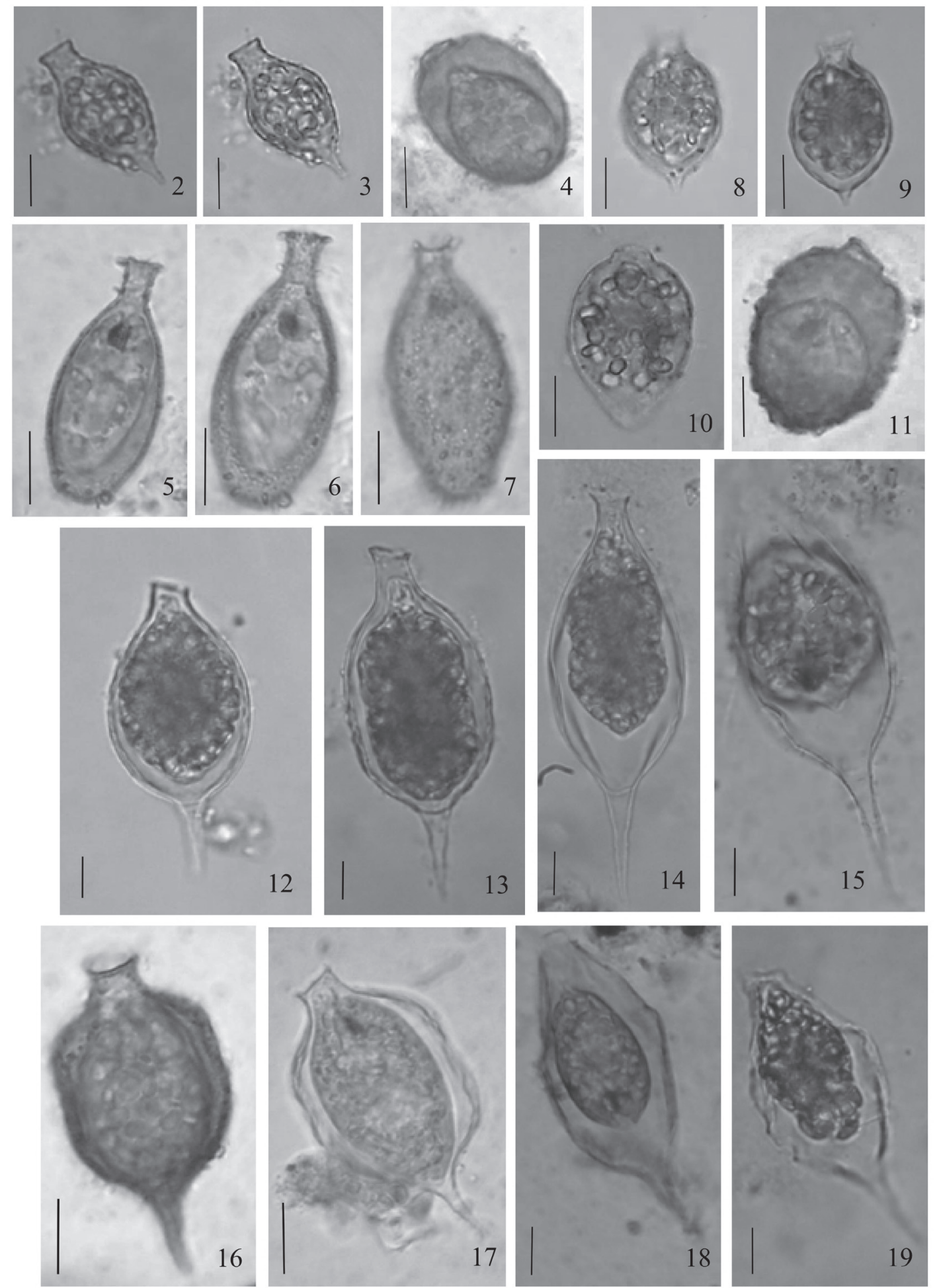

Figuras 2-19. Strombomonas da APA do Ibirapuitã, Rio Grande do Sul, Brasil. 2, 3. Strombomonas asymmetrica. 4. S. borystheniensis var. borystheniensis. 5-7. S. brasiliensis. 8, 9. S. deflandrei var. deflandrei. 10, 11. S. eurystoma f. eurystoma. 12. S. fluviatilis var. levis. 13-15. S. fluviatilis var. rugosa f. major. 16, 17. S. girardiana var. girardiana. 18, 19. S. maxima var. maxima. 19. material fixado. Escala $=10 \mu$ m.

Figures 2-19. Strombomonas of the APA do Ibirapuitã, Rio Grande do Sul State, Brazil. 2, 3. Strombomonas asymmetrica. 4. S. borystheniensis var. borystheniensis. 5-7. S. brasiliensis. 8, 9. S. deflandrei var. deflandrei. 10, 11. S. eurystoma f. eurystoma. 12. S. fluviatilis var. levis. 13-15. S. fluviatilis var. rugosa f. major. 16, 17. S. girardiana var. girardiana. 18, 19. S. maxima var. maxima. 19. fixed material. Scale bar $=10 \mu \mathrm{m}$. 
Apesar de Conforti (1993) ter descrito a espécie dentro do gênero Strombomonas, os indivíduos apresentam espinhos cônicos semelhantes ao gênero Trachelomonas. Entretanto, seu colarinho é indistinto e apresenta aglutinações do meio como os representantes de Strombomonas. Portanto existe necessidade de maiores estudos desse táxon para esclarecer sua posição taxonômica.

Material examinado: BRASIL. Rio GRANDE Do SuL: Livramento, Nascente - E1, 30-XI-2011, S.M. Alvesda-Silva (HAS109261).

Distribuição Geográfica no Brasil: Norte (AM).

Strombomonas deflandrei (Roll) Defl. var. deflandrei, Arch. Protistenk. 69(3): 575, fig. 37. 1930. Figuras 8, 9

Lorica elíptica a ovada, 30-35 $\mu \mathrm{m}$ compr., 20-25 $\mu \mathrm{m}$ larg., $\mathrm{Rc} / 1=1,2-1,7$; vista apical circular; polo anterior com colarinho cilíndrico, curto, reto, às vezes alargado na extremidade distal, $4-5 \mu \mathrm{m}$ compr. , 5-7 $\mu \mathrm{m}$ larg.; polo posterior acuminado abruptamente em processo caudal cônico, reto, 2,5-5 $\mu \mathrm{m}$ compr.; parede levemente granulosa, amarronzada média; cloroplastos numerosos, discoides, ca. $3 \mu \mathrm{m}$ diâm.

Esta espécie apresenta morfologia semelhante a $S$. fluviatilis diferindo pelo processo caudal menor e colarinho alargado na extremidade distal em $S$. deflandrei.

Material examinado: BRASIL. Rio GRANDE do SuL: Alegrete, Banhado Maronna - E9, 23-III-2011, S.M. Alves-da-Silva (HAS109258); Livramento, Passo do Cerrito - E2, 29-III-2012, S.M. Alves-da-Silva (HAS109275).

Distribuição geográfica no Brasil: Norte (AM), Sul (PR, RS).

Strombomonas eurystoma (Stein) Pop. f. eurystoma, Flora plant. Cryptog. 8(1):206, pl. 20, fig. 1-11,13, 1966.

Figuras 10, 11

Lorica amplamente elíptica, 31-34 $\mu \mathrm{m}$ compr., 22-23 $\mu \mathrm{m}$ larg.; $\mathrm{Rc} / 1=1,4-1,5$; vista apical circular; polo anterior termina em colarinho cilindrico, curto, amplo, 2,5-4 $\mu \mathrm{m}$ alt.; 6-7,5 $\mu \mathrm{m}$ larg.; polo posterior levemente acuminado, processo caudal ca. $2 \mu \mathrm{m}$ compr.; parede hialina a castanha média, com aglutinações do meio; cloroplastos discoides, 2,8-3,5 $\mu \mathrm{m}$ diâm.; flagelo ca. de 1/3 do comprimento da lorica.
Este táxon foi citado no Rio Grande do Sul por Alves-da-Silva (2013a) como S. eurystoma var. incurva (Buzenko) Popova, sendo a diferença entre elas é que a última variedade apresenta polo posterior arredondado. Entretanto Brosnan et al. (2005) citam que o desenvolvimento da lorica das Strombomonas ocorre do polo anterior para o posterior, e a lorica pode apresentar morfologia diferenciada de acordo com a ontogenia e do movimento celular durante o seu desenvolvimento. Como todos os indivíduos apresentaram polo posterior acuminado optou-se pela identificação da f. eurystoma, indicando-se a realização de maiores estudos destas duas infraespécies.

Material examinado: BRASIL. Rio GRANDE Do Sul: Livramento, Arroio Sarandizinho - E7, 24-III2011, S.M. Alves-da-Silva (HAS109256); Alegrete, Estância Sabrito - E6, 29-XI-2011, S.M. Alves-daSilva (HAS109270); Alegrete, Estância Sabrito - E6, 27-III-2012, S.M. Alves-da-Silva (HAS109279).

Distribuição geográfica no Brasil: Norte (AM, PA), Sul (RS).

Strombomonas fluviatilis (Lemm.) Defl. var. levis (Lemm.) Skv., Aus der Biol. Sungari Station zur Harbin 1(2): 78, pl. 6, fig. 33. 1925.

Figura 12

Lorica elíptica 64,5-79 $\mu \mathrm{m}$ compr., 25-29 $\mu \mathrm{m}$ larg., $\mathrm{Rc} / 1=2,5-2,7$; polo anterior alargado, terminando em colarinho cilíndrico, levemente enviesado, de 6,4-7,5 $\mu \mathrm{m}$ alt.; 7,5-9, $\mu \mathrm{m}$ larg.; polo posterior acuminado abruptamente em processo caudal cônico, 18-20 $\mu \mathrm{m}$ compr.; parede lisa ou levemente rugosa, amarelada; cloroplastos numerosos, discoides, 3,7-4,6 $\mu \mathrm{m}$ diâm., grãos de paramido numerosos, arredondados ou bastoniformes.

Difere da var. típica da espécie pela maior dimensão celular.

Material examinado: BRASIL. Rio GRANDE do Sul: Livramento, Passo do Cerrito - E2, 24-III-2011, S.M. Alves-da-Silva (HAS 109251); Livramento, Arroio Sarandizinho - E7, 24-III-2011, S.M. Alves-da-Silva (HAS109256); Livramento, Rio Ibirapuitã Chico E10, 25-III-2011, S.M. Alves-da-Silva (HAS109257); Livramento, Nascente - E1, 30-XI-2011, S.M. Alvesda-Silva (HAS109261); Alegrete, Passo da Cancela E5, 29-XI-2011, S.M. Alves-da-Silva (HAS109268); Alegrete, Estância Sabrito - E6, 29-XI-2011, S.M. Alves-da-Silva (HAS109270); Livramento, E8 - Lagoa Marginal Sabrito - 27-III-2012, S.M. Alves-da-Silva (HAS109281). 
Distribuição geográfica no Brasil: Nordeste (PB), Sul (RS, SC).

Strombomonas fluviatialis (Lemm.) Defl. var. rugosa Presc. f. major Yac., Nova Hedwigia 33: 310, pl. XV, fig. 179. 1980.

Figuras 13-15

Lorica elíptica, 83-101 $\mu$ m compr., 28-33 $\mu$ m larg., $\mathrm{Rc} / 1=2,9-3,3$; polo anterior terminado em colarinho cilíndrico, truncado, ligeiramente oblíquo, 8-10 $\mu \mathrm{m}$ alt. e 8-10 $\mu \mathrm{m}$ larg.; polo posterior abruptamente atenuado em processo caudal cônico, reto a ligeiramente curvo 25-33 $\mu \mathrm{m}$ compr.; parede levemente rugosa, amarelada-clara; cloroplastos numerosos, elípticos, 3-4 $\mu \mathrm{m}$ diâm.; haplopirenoides presentes; grãos de paramido numerosos, bastoniformes.

Segundo Yacubson (1980) as dimensões dessa forma são de 98-100 $\mu \mathrm{m}$ compr., $35 \mu \mathrm{m}$ larg., processo caudal de $30 \mu \mathrm{m}$ e colarinho $12 \mu \mathrm{m}$ compr., $9 \mu \mathrm{m}$ larg. No presente estudo foram encontradas duas populações, uma menor com 83-90 $\mu \mathrm{m}$ compr., 20-30 $\mu \mathrm{m}$ larg., $\mathrm{Rc} / 1=2,9-3,3$, processo caudal de 23-25 $\mu \mathrm{m}$, colarinho de 7-11 $\times$ 7-10 $\mu \mathrm{m}$ e outra maior com 92-101 $\mu \mathrm{m}$ compr., 28-33 $\mu \mathrm{m}$ larg., $\mathrm{Rc} / 1=2,9-3,3$; processo caudal $25-33 \mu \mathrm{m}$, colarinho de 8-12 × 7,5-10 $\mu \mathrm{m}$. Apesar das diferenças entre as dimensões os indivíduos analisados possuem morfologia semelhante as descritas pela autora da forma. Muitos exemplares apresentaram uma película transversal na base do processo caudal e presença de haplopirenoides. A observação da película transversal permite a ampliação da diagnose dessa forma.

Material examinado: BRASIL. Rio GRAnde do Sul: Livramento, Passo do Cerrito - E2, 24-III-2011, S.M. Alves-da-Silva (HAS109251); Livramento, Arroio Sarandizinho - E7, 24-III-2011, S.M. Alvesda-Silva (HAS109256); Livramento, Nascente - E1, 30-XI-2011, S.M. Alves-da-Silva (HAS109261); Livramento, Passo do Cerrito - E2, 1-XII-2011, S.M. Alves-da-Silva (HAS109264).

Distribuição geográfica no Brasil: Sul (RS).

Strombomonas girardiana (Playf.) Defl. var. girardiana, Arch. Protistenk. 69(3): 573, figs. 33-35. 1930.

Figuras 16, 17

Lorica subhexagonal, lados quase paralelos, com uma reentrância na região mediana, $42-50 \mu \mathrm{m}$ compr., 19-25 $\mu \mathrm{m}$ larg., $\mathrm{Rc} / 1=2,0-2,2$; vista apical circular; polo anterior terminando em colarinho curto, largo,
3-5 $\mu \mathrm{m}$ alt.; 5-8 $\mu \mathrm{m}$ larg., reto ou obliquo, bordo crenulado; polo posterior atenuado em processo caudal cônico, reto, 5-13 $\mu \mathrm{m}$ compr.; parede castanha clara; cloroplastos arredondados ou elípticos até 3,5 $\mu \mathrm{m}$; haplopirenoides presentes.

No presente estudo muitos indivíduos apresentaram a reentrância mediana da lorica muito tênue como já apontado por Playfair (1915), característica não registrada anteriormente em materiais no Rio Grande do Sul. Mostrando a importância de estudos populacionais desta espécie e cuidado na sua identificação.

Material examinado: BRASIL. Rio GRANDE Do Sul: Livramento, Nascente - E1, 26-III-2011, S.M. Alves-da-Silva (HAS109250); Livramento, Passo do Cerrito - E2, 24-III-2011, S.M. Alves-da-Silva (HAS109251); Livramento, Arroio Sarandizinho E7, 24-III-2011, S.M. Alves-da-Silva (HAS109256); Livramento, Rio Chico Ibirapuitã - E10, 25-III-2011, S.M. Alves-da-Silva (HAS109257); Livramento, Nascente - E1, 30-XI-2011, S.M. Alves-da-Silva (HAS109261); Livramento, Passo do Cerrito - E2, 1-XII-2011, S.M. Alves-da-Silva (HAS109264); Livramento, Arroio Sarandizinho - E7, 1-XII-2011, S.M. Alves-da-Silva (HAS109271); Livramento, Nascente - E1, 28-III-2012, S.M. Alves-da-Silva (HAS109274).

Distribuição geográfica no Brasil: Norte (AM), Sudeste (RJ), Sul (PR, RS, SC).

Strombomonas maxima (Skv.) Defl. var. maxima, Arch. Protistenk. 69(3): 583, figs. 61-65. 1930. Figuras 18, 19

Lorica elíptica, 65,5-78 $\mu \mathrm{m}$ compr., 29-30 $\mu \mathrm{m}$ larg., $\mathrm{Rc} / 1=2,2-2,6$; polo anterior terminado em colarinho cilíndrico, truncado, ligeiramente oblíquo, 3-8,8 $\mu \mathrm{m}$ alt.; 8-10 $\mu \mathrm{m}$ larg.; polo posterior abruptamente atenuado em processo caudal cônico, reto a ligeiramente curvo 15-20 $\mu \mathrm{m}$ compr.; parede lisa, flexível formando pregas longitudinais, amareladaclara, verde a castanha-clara; cloroplastos numerosos, elípticos, ca. 4 um diâm.; haplopirenoides presentes; grãos de paramido numerosos, bastoniformes.

Material examinado: BRASIL. Rio GRANDE Do SuL: Livramento, Passo do Cerrito - E2, 24-III-2011, S.M. Alves-da-Silva (HAS109251); Livramento, Arroio Sarandizinho - E7, 24-III-2011, S.M. Alves-da-Silva (HAS109256); Livramento, Rio Chico Ibirapuitã E10, 25-III-2011, S.M. Alves-da-Silva (HAS109257); Alegrete, Lagoa Marginal Sabrito - E8, 22-III-2011, 
S.M. Alves-da-Silva (HAS 109260); Livramento, Passo do Cerrito - E2, 1-XII-2011, S.M. Alves-da-Silva (HAS 109264); Livramento, Lagoa Marginal Sabrito E8, 29-XI-2011, S.M. Alves-da-Silva (HAS109269); Livramento, Arroio Sarandizinho - E7, 1-XII-2011, S.M. Alves-da-Silva (HAS109271); Livramento, Passo do Cerrito - E2, 29-III-2012, S.M. Alves-da-Silva (HAS 109275); Alegrete, Lagoa Marginal Sabrito E8, 27-III-2012, S.M. Alves-da-Silva (HAS 109281).

Distribuição geográfica no Brasil: Centro-Oeste (MT), Norte (AC, PA, RO), Sudeste (RJ), Sul (PR, RS).

Strombomonas morenensis Bal. \& Dast., Physis 12: 354. 1938.

Figuras 20, 21

Lorica trapezoide, metade posterior mais alargada, 74-77 $\mu \mathrm{m}$ compr., 29-30 $\mu \mathrm{m}$ larg., $\mathrm{Rc} / 1=2,5-2,6$; polo anterior estreita-se abruptamente terminando em colarinho longo, cilíndrico, 13-14 $\mu \mathrm{m}$ compr., com bordo crenulado levemente oblíquo, 5-6 $\mu \mathrm{m}$ larg.; polo posterior atenua-se abruptamente em processo caudal 21-23 $\mu \mathrm{m}$ compr.; parede granulosa, castanhoescura, finamente pontuada; cloroplastos numerosos, discoides, 3-4 $\mu \mathrm{m}$ diâm.

Os exemplares encontrados se apresentaram maiores que os indivíduos encontrados na Argentina, mas com o colarinho menor. Balech \& Dastugue (1938) citam colarinho com comprimento até $19 \mu \mathrm{m}$ e o processo caudal de 19-20 $\mu \mathrm{m}$, enquanto os exemplares na APA possuíam colarinho de 13-14 $\mu \mathrm{m}$ e processo caudal até $23 \mu \mathrm{m}$ compr., mas todas as demais características conferem com a descrição da espécie.

Material examinado: BRASIL. Rio GRANDE Do Sul: Alegrete, Banhado Maronna - E9, 23-III-2011, S.M. Alves-da-Silva (HAS 109258).

Distribuição geográfica no Brasil: Sul (RS).

Strombomonas napiformis (Playf.) Defl. var. brevicollis Playf., Arch. Protistenk. 69(3): 591, figs. 83-84. 1930.

Figuras 22-24

Lorica obotriangular a obovada, 35-67 $\mu \mathrm{m}$ compr., 20-25 $\mu \mathrm{m}$ larg., $\mathrm{Rc} / 1=1,7-3,1$; polo anterior mais alargado que o posterior, terminando rapidamente em colarinho cilíndrico, curto, 2,5-5 $\mu \mathrm{m}$ compr., com bordo alargado levemente oblíquo, 6-9 $\mu \mathrm{m}$ larg.; polo posterior estreita-se abruptamente em um processo caudal longo, levemente curvo, 7-13 $\mu \mathrm{m}$ compr.; parede levemente granulosa, hialina a castanha média; cloroplastos com 8 haplopirenoides; paramidos numerosos, bastoniformes, ca. 1,5 $\mu \mathrm{m}$ compr.

Difere da espécie típica pela forma obovada, pela menor altura do colarinho e pelos cloroplastos com haplopirenoides.

Material examinado: BRASIL. Rio Grande do Sul: Livramento, Nascente - E1, 26-III-2011, S.M. Alvesda-Silva (HAS109250); Livramento, Passo do Cerrito - E2, 24-III-2011, S.M. Alves-da-Silva (HAS109251); Alegrete, Estância Sabrito - E.6, 22-III-2011, S.M. Alves-da-Silva (HAS 109255); Livramento, Arroio Sarandizinho - E7, 24-III-2011, S.M. Alves-da-Silva (HAS109256); Livramento, Rio Chico Ibirapuitã E10, 25-III-2011, S.M. Alves-da-Silva (HAS109257); Alegrete, Banhado Maronna - E9, 23-III-2011, S.M. Alves-da-Silva (HAS 109258); Alegrete, Lagoa Marginal Sabrito - E8, 22-III-2011, S.M. Alves-daSilva (HAS 109260); Livramento, Nascente - E1, 30-XI-2011, S.M. Alves-da-Silva (HAS109261); Livramento, Passo do Cerrito - E2, 1-XII-2011, S.M. Alves-da-Silva (HAS 109264); Livramento, Passo do Ferrão - E3, 2-XI-2011, S.M. Alves-da-Silva (HAS109265); Alegrete, Passo da Cancela - E5, 29XI-2011, S.M. Alves-da-Silva (HAS109268); Alegrete, Lagoa Marginal Sabrito - E8, 29-XI-2011, S.M. Alvesda-Silva (HAS109269); Alegrete, Estância Sabrito E6, 29-XI-2011, S.M. Alves-da-Silva (HAS109270); Livramento, Nascente - E1, 28-III-2012, S.M. Alvesda-Silva (HAS10974); Livramento, Passo do Cerrito E2, 29-III-2012, S.M. Alves-da-Silva (HAS109275); Alegrete, Passo da Cancela - E5, 27-III-2012, S.M. Alves-da-Silva (HAS 109278); Livramento, Arroio Sarandizinho - E7, 29-III-2012, S.M. Alves-da-Silva (HAS 109280).

Distribuição geográfica no Brasil: Sul (RS).

Strombomonas ovalis (Playf.) Defl. var. ovalis, Arch. Protistenk. 69(3): 577, fig. 42-43. 1930.

Figuras 25, 26

Lorica ovada 38-45 $\mu \mathrm{m}$ compr., $23 \mu \mathrm{m}$ larg., $\mathrm{Rc} / 1=1,6-2$; polo anterior com colarinho cilíndrico curto, bordo liso ou crenulado; 2,5-5,0 $\mu \mathrm{m}$ alt., 5-7,6 $\mu \mathrm{m}$ larg.; polo posterior estreita-se em processo caudal cônico, reto ou levemente curvo, 6,3-7,6 $\mu \mathrm{m}$ compr.; parede lisa ou com pequenos grânulos; cloroplastos discoides, 3,0-3,8 $\mu \mathrm{m}$ de diâm.

Material examinado: BRASIL. Rio GRANDE do Sul: Livramento, Rio Chico Ibirapuitã - E10, 25-III-2011, 

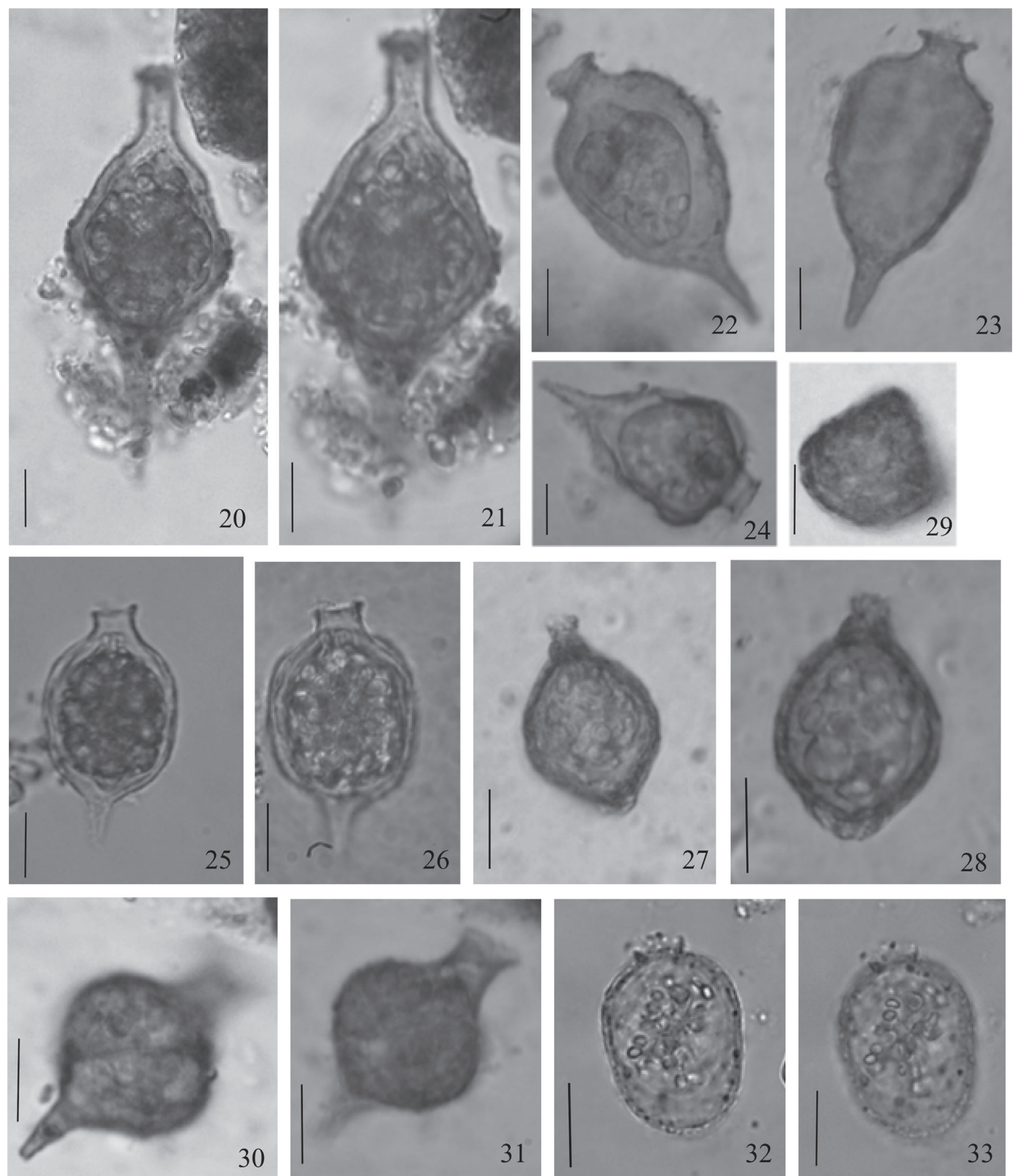

Figuras 20-33. Strombomonas da APA do Ibirapuitã, Rio Grande do Sul, Brasil. 20-21. S. morenensis. 22-24. S. napiformis var. brevicollis. 25, 26. S. ovalis var. ovalis. 27-29. S. recurvata. 29. vista apical. 30, 31. S. rotunda var. rotunda. 32, 33. S. scabra comb. nov. Escala $=10 \mu \mathrm{m}$.

Figures 20-33. Strombomonas of the APA do Ibirapuitã, Rio Grande do Sul State, Brazil. 20-21. S. morenensis. 22-24. S. napiformis var. brevicollis. 25, 26. S. ovalis var. ovalis. 27-29. S. recurvata. 29. apical view. 30, 31. S. rotunda var. rotunda. 32, 33. S. scabra comb. nov. Scale bar $=10 \mu \mathrm{m}$. 
S.M. Alves-da-Silva (HAS109257); Livramento, Nascente - E1, 30-XI-2011, S.M. Alves-da-Silva (HAS109261); Livramento, Passo do Ferrão - E3, 2-XI-2011, S.M. Alves-da-Silva (HAS109265); Alegrete, Passo da Cancela - E5, 29-XI-2011, S.M. Alves-da-Silva (HAS109268); Alegrete, Lagoa Marginal Sabrito - E8, 29-XI-2011, S.M. Alves-daSilva (HAS109269); Livramento, Passo do Cerrito E2, 29-III-2012, S.M. Alves-da-Silva (HAS109275).

Distribuição geográfica no Brasil: Norte (AM, RO), Sudeste (RJ, ES), Sul (PR, RS).

Strombomonas recurvata Conf. \& Joo, Cryptogamie. Algol. 15(4): 277, fig. 76 a-g. 1994.

Figuras 27-29

Lorica ovada, 26,5-30,2 $\mu \mathrm{m}$ compr., 18-20 $\mu \mathrm{m}$ larg., $\mathrm{Rc} / 1=1,4-1,5$; vista polar triangular com ângulos arredondados; vista lateral de elíptica a triangular; polo anterior termina em colarinho cilíndrico, com espessamento na sua base, ca. $5 \mu \mathrm{m}$ alt., 4,5-5 $\mu \mathrm{m}$ larg.; polo posterior levemente acuminado em processo caudal mamilado irregular; parede espessa, castanho-avermelhada; cloroplastos com duplopirenoides.

Material examinado: BRASIL. Rio GRANDE Do SuL: Livramento, Nascente - E1, 30-XI-2011, S.M. Alvesda-Silva (HAS109261).

Distribuição geográfica no Brasil: Sul (RS).

Strombomonas rotunda (Playf.) Defl. var. rotunda,

Arch. Protistenk. 69(3): 593, fig. 88, 1930.

Figuras 30, 31

Lorica com região mediana transversalmente elíptica, 35-38 $\mu \mathrm{m}$ compr., 17-18 $\mu \mathrm{m}$ larg., $\mathrm{Rc} / 1=1,6-1,7$; vista apical circular; polo anterior atenuado abruptamente em colarinho cilíndrico, com bordo reto ou levemente oblíquo, liso ou crenulado, com 5-6,4 $\mu \mathrm{m}$ alt., 6-7,0 $\mu \mathrm{m}$ larg.; polo posterior abruptamente atenuado em processo caudal agudo, reto, de 6-10 $\mu \mathrm{m}$ compr.; parede lisa, castanho-clara a avermelhada; cloroplastos numerosos discoides, ca. $5 \mu \mathrm{m}$ diâm.; grãos de paramido numerosos, elípticos ca. $3 \mu \mathrm{m}$ diâm.; flagelo ca. uma vez o compr. da lorica.

Material examinado: BRASIL. Rio GRANDE Do Sul: Livramento, Arroio Sarandizinho - E7, 24-III-2011, S.M. Alves-da-Silva (HAS109256); Alegrete, Lagoa Marginal Sabrito - E8, 22-III-2011, S.M. Alves-daSilva (HAS 109260); Livramento, Nascente - E1,
30-XI-2011, S.M. Alves-da-Silva (HAS109261); Alegrete, Passo da Cancela - E5, 27-III-2012, S.M. Alves-da-Silva (HAS 109278); Alegrete, Estância Sabrito - E6, 27-III-2012, S.M. Alves-da-Silva (HAS109279).

Distribuição geográfica no Brasil: Nordeste (PB), Norte (AM, PA), Sul (RS, SC), Sudeste (RJ, SP).

Strombomonas scabra (Playf.) S. M. Alves-da-Silva comb. nov. Basionnimo: Trachelomonas scabra Playf., Proc.Linn. Soc. New South Wales 40:28, pl. IV, fig. 2, 3. 1915.

Figuras 32, 33

Lorica amplamente elíptica, 28-30 $\mu \mathrm{m}$ compr., 19-21 $\mu \mathrm{m}$ larg., $\mathrm{Rc} / 1=1,4-1,6$; polo anterior abruptamente atenuado, terminando em colarinho curto, baixo, bordo crenulado, 3-4,5 $\mu \mathrm{m}$ alt., 7,6 $\mu \mathrm{m}$ larg.; polo posterior ligeiramente acuminado, arredondado, sem processo caudal; parede espessa, rugosa, castanho-claro a escura; cloropastos numerosos, discoides, ca. $3 \mu \mathrm{m}$.diâm.; haplopirenoides presentes; flagelo até duas vezes o comprimento da lorica.

Tell \& Conforti em 1986 transferiram Trachelomonas scabra Playfair para Strombomonas sob a denominação de S. scabra (Playf.) Tell \& Conf. em Bibliotheca Phycologica 75:170. Os autores indicaram o basiônimo entre parênteses logo em seguida do nome combinado, mas não esclareceram tratar-se de uma combinação nova. Por esta razão a proposta de Tell e Conforti (1986) não é válida. Em 1988 estes mesmos autores publicam S. scabra (Playf.) Tell e Conf. var. scabra em Nova Hedwigia 46:545, junto com várias outras combinações, mas novamente não indicam a espécie típica ser uma combinação nova, continuando esta espécie invalida. Concorda-se com a transferência efetuada por Tell \& Conf. de Trachelomonas scabra Playf. para Strombomonas, visto que a lorica possui a capacidade de aglutinar na sua superfície partículas do meio (Conforti et al. 1994, Brosnan et al., 2005), e apresentar terminação do colarinho indistinto. Este táxon tem sido amplamente citado no país como S. scabra (Playf.) Tell \& Conf.

Material examinado: BRASIL. Rio Grande do Sul: Livramento, Nascente - E1, 26-III-2011, S.M. Alvesda-Silva (HAS109250); Alegrete, Passo da Cancela E5, 29-XI-2011, S.M. Alves-da-Silva (HAS109268); Alegrete, Estância Sabrito - E6, 29-XI-2011, S.M. Alves-da-Silva (HAS109270); Livramento, Passo do Cerrito - E2, 29-III-2012, S.M. Alves-da-Silva (HAS109275). 
Distribuição no Brasil: Norte (AM, PA), Centro-oeste (MT), Sudeste (RJ, SP), Sul (PR, RS).

Strombomonas tetraptera Bal. \& Dast. var. gallica Bourr. \& Couté, Rev. Algol. 13(4): 299, fig. 17. 1978. Figuras 34-39

Lorica de contorno geral elíptico, torção helicóide, 28-47 $\mu \mathrm{m}$ compr., 20-23 $\mu \mathrm{m}$ larg., Rc/1 = 1,4-2,0; vista polar quadrangular, lados côncavos, ângulos arredondados; polo anterior alongado, atenuado em colarinho cilíndrico, curto, levemente oblíquo, 2,5-5 $\mu \mathrm{m}$ alt.; 6-8,8 $\mu \mathrm{m}$ larg.; polo posterior atenuado abruptamente em processo caudal, 2,5-7,6 $\mu \mathrm{m}$ compr.; parede irregularmente granulosa, hialina a esverdeada; cloroplastos discoides ou elípticos, numerosos, 2,5-3 $\mu \mathrm{m}$ de diâm.

Segundo Bourrelly \& Couté (1978) esta variedade difere da espécie típica pela torção regular helicóide da lorica, sem expansões aliformes marcante, pelas menores dimensões $(32-40 \times 18-20 \mu \mathrm{m})$, pela vista apical quadrangular com lados côncavos e ângulos arredondados. Os indivíduos observados apresentaram dimensões maiores que a var. gallica, próximas da espécie típica, mas vista apical semelhante a var. gallica e torção levemente helicoide. Foi observado também em alguns indivíduos retração lateral da lorica após fixação, e maiores dimensões do processo caudal dependendo da posição em que o indivíduo é observado.

Estudos envolvendo biologia molecular com as duas variedades necessitam ser realizados para esclarecer se a variedade gallica não é uma expressão morfológica da espécie típica.

Material examinado: BRASIL. Rio GRANDE Do Sul: Alegrete, Passo da Cancela - E5, 22-III-2011, S.M. Alves-da-Silva (HAS109254); Alegrete, Estância Sabrito - E6, 22-III-2011, S.M. Alves-da-Silva (HAS 109255); Livramento, Arroio Sarandizinho - E7, 24-III-2011, S.M. Alves-da-Silva (HAS109256); Livramento, Rio Chico Ibirapuitã - E10, 25-III-2011, S.M. Alves-da-Silva (HAS109257); Alegrete, Lagoa Marginal Sabrito - E8, 22-III-2011, S.M. Alves-daSilva (HAS 109260); Livramento, Nascente - E1, 30-XI-2011, S.M. Alves-da-Silva (HAS109261); Livramento, Passo do Cerrito - E2, 1-XII-2011, S.M. Alves-da-Silva (HAS 109264); Livramento, Passo do Ferrão - E3, 2-XI-2011, S.M. Alves-da-Silva (HAS109265); Alegrete, Passo da Cancela - E5, 29-XI-2011, S.M. Alves-da-Silva (HAS109268); Alegrete, Estância Sabrito - E6, 29-XI-2011, S.M.
Alves-da-Silva (HAS109270); Livramento, Passo do Cerrito - E2, 29-III-2012, S.M. Alves-da-Silva (HAS109275); Livramento, Passo do Ferrão - E3, 30-III-2012, S.M. Alves-da-Silva (HAS109276).

Distribuição geográfica no Brasil: Centro-oeste (MTcitada como $S$. tetraptera por Menezes \& Fernandes 1987), Norte (AM) e Sul (RS).

Strombomonas triquetra (Playf.) Defl. var. torta Rino, Rev. Ciênc. Biol. 5: 169, pl. 9, figs 7-10. 1972.

Figuras 40, 41

Lorica levemente triangular a trapezoidal, 33$43 \mu \mathrm{m}$ compr., 18-21 $\mu \mathrm{m}$ larg., Rc/1 = 1,7-2,2; com lados retilíneos ou levemente constrictos; vista apical triangular com ângulos arredondados; polo anterior abruptamente atenuado em colarinho truncado, oblíquo, largo, bordo crenulado ca. 2,5-4 $\mu \mathrm{m}$ alt., 5,5-9 $\mu \mathrm{m}$ larg.; polo posterior abruptamente atenuado em processo caudal cônico, 1,5-4 $\mu \mathrm{m}$ compr.; parede granulosa, castanho-claro a castanho-médio; cloroplastos numerosos, discoides, $3 \mu \mathrm{m}$ diâm., haplopirenoides presentes.

Material examinado: BRASIL. Rio GRANDE Do Sul: Livramento, Nascente - E1, 26-III-2011, S.M. Alves-da-Silva (HAS109250); Livramento, Passo do Cerrito - E2, 24-III-2011, S.M. Alves-da-Silva (HAS109251); Livramento, Passo do Ferrão - E3, 25-III-2011, S.M. Alves-da-Silva (HAS109252); Alegrete, Passo da Cancela - E5, 22-III-2011, S.M. Alves-da-Silva (HAS109254); Alegrete, Estância Sabrito - E6, 22-III-2011, S.M. Alves-da-Silva (HAS 109255); Livramento, Arroio Sarandizinho - E7, 24-III-2011, S.M. Alves-da-Silva (HAS109256); Livramento, Rio Chico Ibirapuitã - E10, 25-III-2011, S.M. Alves-da-Silva (HAS109257); Alegrete, Banhado Maronna - E9, 23-III-2011, S.M. Alves-da-Silva (HAS 109258); Alegrete, Lagoa Marginal Sabrito - E8, 22-III-2011, S.M. Alves-da-Silva (HAS 109260); Livramento, Passo do Cerrito - E2, 1-XII-2011, S.M. Alves-da-Silva (HAS 109264); Alegrete, Estância Maronna - E4, 29-XI-2011, S.M. Alves-da-Silva (HAS109266); Alegrete, Passo da Cancela - E5, 29XI-2011, S.M. Alves-da-Silva (HAS109268); Alegrete, Estância Sabrito - E6, 29-XI-2011, S.M. Alves-daSilva (HAS109270); Livramento, Passo do Cerrito - E2, 29-III-2012, S.M. Alves-da-Silva (HAS109275); Alegrete, Estância Maronna - E4, 27-III-2012, S.M. Alves-da-Silva (HAS109277); Alegrete, Passo da Cancela - E5, 27-III-2012, S.M. Alves-da-Silva (HAS 

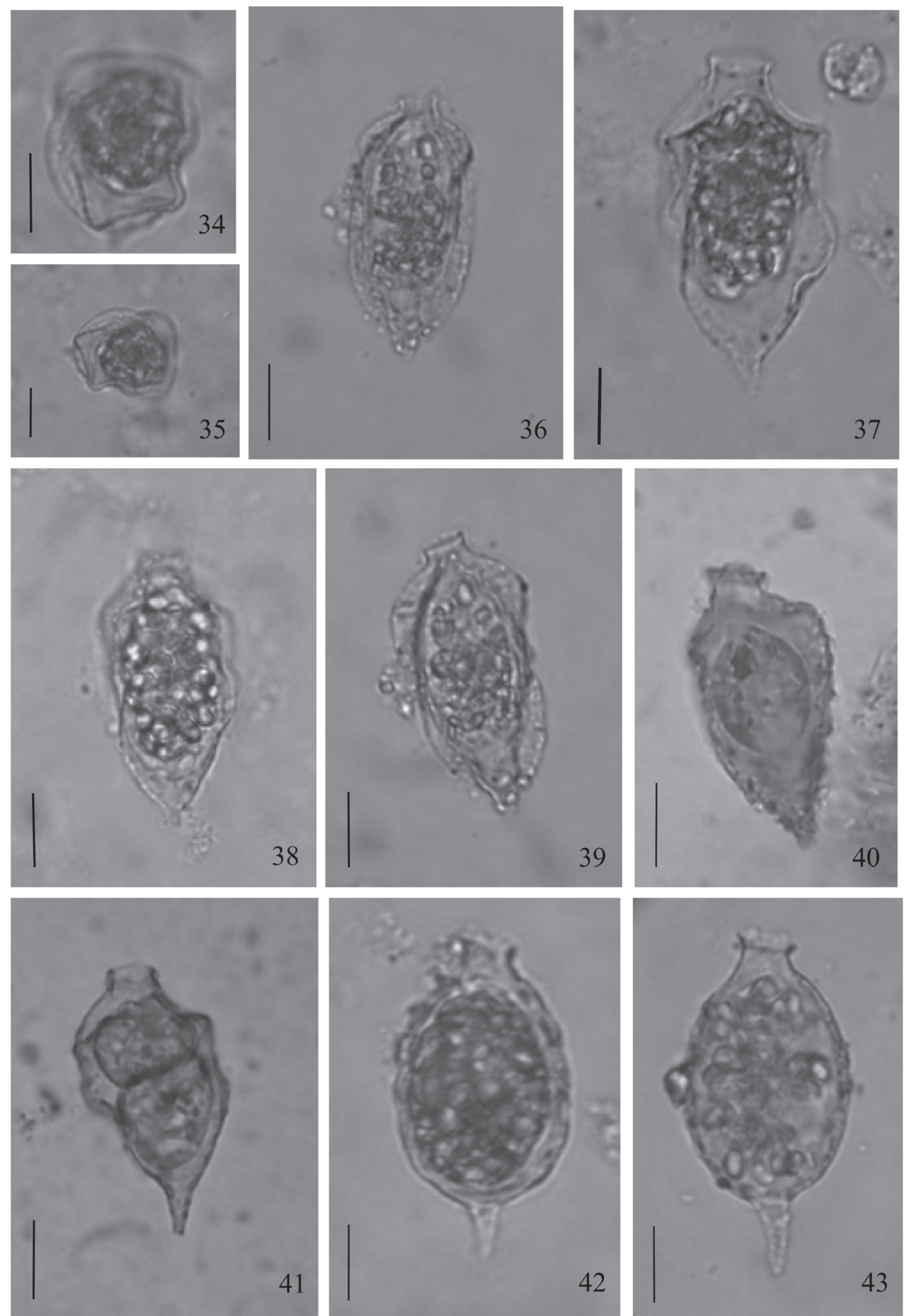

Figuras 34-43. Strombomonas da APA do Ibirapuitã, Rio Grande do Sul, Brasil. 34-39. S. tetraptera var. gallica. 34, 35. vista apical. 40, 41. S. triquetra var. torta. 41. célula em reprodução. 42, 43. S. verrucosa var. verrucosa. Escala $=10 \mu \mathrm{m}$.

Figures 34-43. Strombomonas of the APA do Ibirapuitã, Rio Grande do Sul State, Brazil. 34-39. S. tetraptera var. gallica. 34, 35. apical view. 40, 41. S. triquetra var. torta. 41. cell reproduction. 42, 43. S. verrucosa var. verrucosa. Scale bar $=10 \mu \mathrm{m}$. 
109278); Alegrete, Estância Sabrito - E6, 27-III-2012, S.M. Alves-da-Silva (HAS109279); Livramento, Arroio Sarandizinho - E7, 29-III-2012, S.M. Alvesda-Silva (HAS 109280).

Distribuição geográfica no Brasil: Nordeste (PB), Sul (RS).

Strombomonas verrucosa (Daday) Defl. var. verrucosa, Arch. Protistenk. 69(3): 566, fig. 6-10. 1930.

Figuras 42, 43

Lorica ovada a trapezoidal, 38-54 $\mu \mathrm{m}$ compr., 18,5-25 $\mu \mathrm{m}$ larg., $\mathrm{Rc} / 1=1,9-2,2$; vista apical circular; polo anterior atenuado abruptamente em colarinho cilíndrico, com bordo reto ou levemente oblíquo, liso ou crenulado, com 2,5-6 $\mu \mathrm{m}$ alt., 6,5-8 $\mu \mathrm{m}$ larg.; polo posterior abruptamente atenuado em processo caudal conico, reto, 8-13 $\mu \mathrm{m}$ compr.; parede granulosa, castanho-media a escura; cloroplastos numerosos discoides, ca. $5 \mu \mathrm{m}$ diâm., haplopirenoides presentes; flagelo ca. uma vez o comprimento da lorica.

Strombomonas verrucosa (Daday) Defl. é uma espécie polimórfica originando considerável confusão na sua identificação taxonômica (Alves-da-Silva \& Bicudo 2006). Os indivíduos encontrados poderiam ser identificados como a var. zmiewika, mas optou-se em identificar-se como a var. típica devido a plasticidade fenotípica podendo as categorias infraespecíficas descritas representarem expressões morfológicas de um único táxon (Menezes 1992 e Alves-da-Silva \& Bicudo 2006).

Material examinado: BRASIL, Rio GRANDE Do Sul: Livramento, Nascente - E1, 26-III-2011, S.M. Alves-da-Silva (HAS109250); Livramento, Passo do Cerrito - E2, 24-III-2011, S.M. Alves-da-Silva (HAS109251); Livramento, Passo do Ferrão - E3, 25-III-2011, S.M. Alves-da-Silva (HAS109252); Alegrete, Estância Maronna - E4, 23-III-2011, S.M. Alves-da-Silva (HAS 109253); Alegrete, Passo da Cancela - E5, 22-III-2011, S.M. Alves-da-Silva (HAS109254); Alegrete, Estância Sabrito - E6, 22-III-2011, S.M. Alves-da-Silva (HAS 109255); Livramento, Arroio Sarandizinho - E7, 24-III-2011, S.M. Alves-da-Silva (HAS109256); Livramento, Rio Chico Ibirapuitã - E10, 25-III-2011, S.M. Alves-daSilva (HAS109257); Livramento, Nascente - E1, 30-XI-2011, S.M. Alves-da-Silva (HAS109261); Livramento, Passo do Cerrito - E2, 1-XII-2011, S.M. Alves-da-Silva (HAS 109264); Livramento, Passo do Ferrão - E3, 2-XI-2011, S.M. Alves-da-Silva
(HAS109265); Alegrete, Estância Maronna - E4, 29-XI-2011, S.M. Alves-da-Silva (HAS109266); Alegrete, Passo da Cancela - E5, 29-XI-2011, S.M. Alves-da-Silva (HAS109268); Alegrete, Estância Sabrito - E6, 29-XI-2011, S.M. Alves-da-Silva (HAS109270); Livramento, Arroio Sarandizinho E7, 1-XII-2011, S.M. Alves-da-Silva (HAS 109271); Livramento, Nascente - E1, 28-III-2012, S.M. Alvesda-Silva (HAS10974); Alegrete, Estância Maronna E4, 27-III-2012, S.M. Alves-da-Silva (HAS109277); Alegrete, Passo da Cancela - E5, 27-III-2012, S.M. Alves-da-Silva (HAS 109278); Alegrete, Estância Sabrito - E6, 27-III-2012, S.M. Alves-da-Silva (HAS109279); Livramento, Arroio Sarandizinho E7, 29-III-2012, S.M. Alves-da-Silva (HAS 109280).

Distribuição geográfica no Brasil: Norte (AM, PA), Centro-Oeste (MS, MT), Nordeste (MA), Sudeste (RJ), Sul (PR, RS, SC).

O resultado do presente estudo do gênero foi semelhante aos já encontrados por Alves-daSilva \& Klein (2015) para o gênero Phacus, ou seja, Strombomonas ocorreu tanto na comunidade fitoplanctônica como na perifítica, sendo registrado maior riqueza em locais com macroalgas submersas (Chara sp. e Nitella sp.) e macrófitas aquáticas. Esses vegetais podem formar um microhabitat rico em nutrientes como amônia, fósforo e matéria orgânica, que favorece a presença das Euglenophyta (Rodrigues \& Bicudo 2001, Alves-da-Silva \& Bicudo 2003, Conforti \& Zalocar 2003, Salazar 2004 e Alves-daSilva \& Kurrle 2009).

Houve diferente distribuição da riqueza específica (S) que variou de um a 11 táxons por amostra, com as maiores ocorrendo no outono e primavera/2011, e a menor riqueza no outono/2012 (tabela 1).

Os locais com maiores riquezas foram: E1(11 táxons), E2 (9 e 8 táxons), E5 (8 táxons), E6 (9 táxons), E7(10 táxons) e E10 (9 táxons). Nestes locais as variáveis ambientais foram baixíssima profundidade $(0,1-0,52 \mathrm{~m})$ e visualmente baixa correnteza, devido à estiagem ocorrida no período, a temperatura da água alta $\left(19-31^{\circ} \mathrm{C}\right)$, o $\mathrm{pH}$ levemente ácido a levemente alcalino (6,3-7,3), condutividade elétrica de $66-131 \mu \mathrm{S} \mathrm{cm}^{-1}$, matéria orgânica média a alta (5,6-9,2 $\left.\mathrm{mg} \mathrm{L}^{-1}\right)$ e sílica alta (21-26 $\left.\mathrm{mg} \mathrm{L}^{-1}\right)$.

Quanto à temperatura da água muitos espécimes de Strombomonas podem ser considerados como euritérmicos, por ocorrerem em amplitude de temperatura superior a $10^{\circ} \mathrm{C}$, sendo alguns estenotérmicos (Alves-da-Silva \& Bridi 2004, Alvesda-Silva \& Bicudo 2006). 
Segundo Alves-da-Silva \& Torres (1994), Alvesda-Silva \& Bridi (2004), Alves-da-Silva \& Bicudo (2006), Araújo et al. (2012) e Alves-da-Silva et al. (2013a) muitos táxons de Strombomonas toleram uma ampla variação de $\mathrm{pH}$, ocorrendo em águas levemente ácidas a alcalinas como ocorreu no presente estudo.

Alves-da-Silva \& Kurrle (2009) concluíram que este gênero apresenta ampla tolerância quanto à condutividade, visto que Conforti (1993) registrou o maior número de táxons de Strombomonas em $762 \mu \mathrm{S} \mathrm{cm}^{-1}$, enquanto Zaburlin et al. (2004) registraram táxons entre $34-49 \mu \mathrm{S} \mathrm{cm}^{-1}$. No estudo as maiores riquezas ocorreram em condutividade elétrica entre $66-131 \mu \mathrm{S} \mathrm{cm}^{-1}$, valores próximos aos encontrados por Alves-da-Silva et al. (2013a) de 13,2-113,6 $\mu \mathrm{S} \mathrm{cm}^{-1}$, em um arroio raso no Rio Grande do Sul quando também foram registradas as maiores riquezas. Alves-da-Silva \& Bicudo (2006) ao estudarem um reservatório raso no RS, registraram maiores riquezas em condutividades por volta de $100 \mu \mathrm{S} \mathrm{cm}^{-1}$. Estes três últimos estudos indicam que apesar da ampla tolerância à condutividade, os táxons parecem ter preferência por baixas condutividades até concentrações ca. $130 \mu \mathrm{S} \mathrm{cm}^{-1}$.

A matéria orgânica registrada na APA caracterizou condições de poluição orgânica mediana (mesossapróbicas), segundo classificação de Hamm (1969), condições já encontradas por Conforti \& Pérez (2000) e Alves-da-Silva et al. (2007, 2013a) em estudos abrangendo Strombomonas, embora espécies do gênero já tenham sido registradas em águas com a alta concentração de matéria orgânica (Cecy 1990, Zuberlin et al. 2004, Domingues \& Torgan 2011).

Os teores de sílica nos locais com maiores riquezas foram altas (21-26 $\left.\mathrm{m} \mathrm{L}^{-1}\right)$ o que deve ter propiciado a presença do gênero, resultados semelhantes aos registrados por Alves-da-Silva \& Bridi (2004).

Apesar dos táxons do gênero apresentarem ampla distribuição por ter ocorrido representantes em todos locais amostrados, a frequência de ocorrência dos táxons foi baixa (tabela 1), pois $72 \%$ foram pouco frequentes ou raros ( 8 e 5 táxons, respectivamente). Somente $S$. verrucosa var. verrucosa foi muito frequente na área, sendo Strombomonas borystheniensis var. borystheniensis, S. napiformis var. brevicollis. S. tetraptera var. gallica, S. triquetra var. torta frequentes.

Strombomonas verrucosa var. verrucosa é uma espécie cosmopolita e apresenta ampla distribuição no Brasil, sendo referida como extremamente polimórfica. Na APA foi o táxon melhor representado no estudo, ocorrendo em $77 \%$ do total de amostras. Novos estudos moleculares são necessários para confirmar a existência de infraespécies dessa espécie.

Destaca-se a ocorrência de $S$. maxima var. maxima, $S$. rotunda var. rotunda, S. scabra em quatro regiões do Brasil.

É proposta uma nova combinação Strombomonas scabra comb. nov. para validar esta espécie, pois, desde a proposta de Tell \& Conforti (1986), esta espécie tem sido citada em vários artigos como Strombomonas scabra nome não valido, visto que não obedece ao atual Código Internacional de Nomenclatura de Algas, Fungos e Plantas (Código de Melbourne).

Existe necessidade de maiores estudos morfológicos e moleculares para esclarecer a posição taxonômica de Strombomonas brasiliensis, visto que a espécie apresenta características morfológicas dos gêneros Trachelomonas e Strombomonas.

Todas as espécies e infraespécies de Strombomonas são novos registros para o sudoeste do Rio Grande do Sul. Destaca-se $S$. brasiliensis por ser o primeiro registro para o Estado e o segundo para o país e $S$. recurvata, citada pela segunda vez no Brasil. S. morenensis e $S$. napiformis var. brevicollis são registrados pela segunda vez no Rio Grande do Sul e Brasil. Todos estes táxons são muito raros em nível mundial.

É essencial que novos estudos morfológicos em ambientes naturais sejam realizados, assim como ampliado o número de táxons de Strombomonas em estudos moleculares, devido à alta plasticidade morfológica existente dentro do gênero e em especial em alguns táxons. Deve-se considerar o fato de que, segundo Brosnan et al. (2005), durante o desenvolvimento da célula a lorica pode sofrer modificações, podendo apresentar diferentes contornos dentro da mesma espécie.

Este estudo permitiu suprir em parte a lacuna existente na distribuição dessas microalgas no sudoeste do Bioma Pampa. Salienta-se que novos estudos devem ser incentivados nessa e em outras regiões do Estado, com o objetivo de ampliar o conhecimento taxonômico e ecológico de espécies do gênero Strombomonas ainda pouco conhecido em nível nacional e mundial.

\section{Agradecimentos}

Ao Conselho Nacional de Desenvolvimento Científico e Tecnológico pelas Bolsas de Iniciação Científica concedida às acadêmicas Isabele Corino 
Klein (Proc.125705/2015-8) e Isadora Lieske (Processo 117026/16-6). Aos funcionários do Instituto Chico Mendes de Conservação da Biodiversidade Eridiane Lopes da Silva (gestora da APA do Ibirapuitã) e ao Raul Paixão Coelho pelo apoio logístico durante a coleta das amostras. À Fundação Zoobotânica do Rio Grande do Sul pela infraestrutura. A todos os colegas do Museu de Ciências Naturais que auxiliaram nas expedições de coleta, análises químicas e confecção do mapa. Ao Dr. Prof. Carlos Eduardo de Mattos Bicudo pelo valioso auxílio.

\section{Literatura citada}

Alves-da-Silva, S.M. \& Avila, I.R. 1997. Levantamento florístico da Euglenaceae pigmentadas do Parque Estadual Delta do Jacuí, Rio Grande do Sul, Brasil. Iheringia, Série Botânica 48: 85-102.

Alves-da-Silva, S.M. \& Bicudo, C.E.M. 2003. Strombomonas carinata sp. nov. and Strombomonas ferrazi sp. nov., two new Euglenophyceae from the state of Rio Grande do Sul, Southern Brazil. Acta Botanica Brasilica 17: 147-154.

Alves-da-Silva, S.M. \& Bicudo, C.E.M. 2006. Contribution to the knowledge of genus Strombomonas (Euglenophyceae) of the state of Rio Grande do Sul, southern Brazil. Algological Studies 119: 29-58.

Alves-da-Silva, S.M. \& Bridi, F.C. 2004. Euglenophyta no Parque Estadual Delta do Jacuí, Rio Grande do Sul, Sul do Brasil. 3. Gênero Strombomonas Defl. Acta Botanica Brasilica 18: 555-572.

Alves-da-Silva, S.M. \& Crossetti, L.O. 1999. Novas citações de ocorrência de Euglenaceae pigmentadas para o estado do Rio Grande do Sul, Brasil. Hoehnea 26: 47-60.

Alves-da-Silva, S.M. \& Hahn, A.T. 2001. Lista das Euglenophyta registradas em ambientes de águas continentais e costeiras do Estado do Rio Grande do Sul, Brasil. Iheringia, Série Botânica 55: 171-188.

Alves-da-Silva, S.M. \& Klein, I.C. 2015. Euglenophyceae na Área de Proteção Ambiental do Rio Ibirapuitã, sudoeste do Estado do Rio Grande do Sul, Brasil. 1. Cryptoglena Marin \& Melkonian emend. Kosmala \& Zakrýs, Monomorphina (Ehrenberg) Mereschkowsky emend. Kosmala \& Zackýs e Phacus Dujardin. Hoehnea 42: 471-496.

Alves-da-Silva, S.M. \& Kurrle, I.L.P.V. 2009. O gênero Strombomonas (Euglenophyceae pigmentadas) em ambientes lênticos na Planície Costeira do Rio Grande do Sul, Brasil. Acta Botanica Brasilica 23: 944-955.

Alves-da-Silva, S.M. \& Menezes, M. 2015. Euglenophyceae in Lista de Espécies da Flora do Brasil. Jardim Botânico do Rio de Janeiro. Disponivel em http://floradobrasil. jbrj.gov.br/jabot/floradobrasil/FB108841 (acesso em 26-I-2017).
Alves-da-Silva, S.M. \& Tamahana, M. da S. 2008. Ocorrência de Euglenophyceae pigmentadas em rizipiscicultura na Região do Vale do Itajaí, SC, Sul do Brasil. Acta Botanica Brasilica 22: 145-163.

Alves-da-Silva, S.M. \& Torres, J.R. 1994. O gênero Strombomonas (Euglenaceae) no Parque Zoológico, Sapucaia do Sul e do Jardim Botânico de Porto Alegre, Rio Grande do Sul, Brasil. Iheringia, Série Botânica 44: 15-32.

Alves-da-Silva, S.M., Escobar, K.C. \& Juliano, V.B. 2015. Novos registros de Trachelomonas Ehr. emend. Defl. (Euglenophyceae) para o Estado do Rio Grande do Sul e para o Brasil. Hoehnea 43: 1-10.

Alves-da-Silva, S.M., Hermany, F. \& Oliveira, M.A. 2007. Diversity and ecological considerations on pigmented Euglenophyceae the State Parque of the Jacuí Delta, Rio Grande do Sul, southern Brazil. Biociências 15: 8-20.

Alves-da-Silva, S.M., Cabreira J. da C., Consoni\& K.F. \& Lobo, E.A. 2013a. Gênero Strombomonas Deflandre (Euglenophyceae pigmentadas em ambiente lótico subtropical, município de Triunfo, RS, Brasil: riqueza e distribuição geográfica. Hoehnea 40: 381-402.

Alves-da-Silva, S.M., Cabreira J. da C., Voss, J.G. \& Lobo, E.A. 2013b. Species richness of the genera Trachelomonas and Strombomonas (pigmented Euglenophyceae) in a subtropical urban lake in the Porto Alegre Botanical Garden, RS, Brazil. Acta Botanica Brasilica 27: 464-475.

APHA - American Public Health Association. 2005. Standard methods for the examination of water and wasterwater. American Public Health Associations, Washington.

Araújo, G.J.M., Barbosa, J.E. de L. \& Barbosa, L.G. 2012. Pigmented Euglenophytes in a natural and shallow lake in the semiarid region of Paraíba State, Brazil. Brazilian Journal of Botany 35: 17-30.

Balech, E. \& Dastugue, C.E. 1938. Nota preliminar sobre Strombomonas y Trachelomonas. Physis 12: 354-357.

Bicudo, C.E. de M. \& Menezes, M. 2005. Gênero de algas de águas continentais do Brasil: chave para identificação e descrições. 1 ed. Rima, São Carlos.

Bicudo, C.E. de M. \& Menezes, M. 2016. Phylogeny and classification of Euglenophyceae: A brief review. Frontier in Ecology and Evolution 4: 1-15.

Bittencourt-Oliveira, M. 1997. Euglenophyceae do Reservatório de Balbina, Estado do Amazonas, Brasil. Hoehnea 24: 21-35.

Bourrelly, P.C. 1970. Les algues d'eau douce: initation à la systematique, III. Les algues blues et rouges, les Eugléniens, Peridiniens et Cryptomonadines. Éditions N. Boubée, Paris.

Bourrelly, P.C. \& Couté, A. 1978. Alges d'eau douce rares ou nouvelles pour la flore française. Revue Algologique 13: 295-307. 
Brosnan, S., Brow, P.J.P., Farmer, M.A. \& Triemer, R.E. 2005. Morphological separation of the Euglenoid genera Trachelomonas and Strombomonas (Euglenophyta) based on lorica development and posterior strip reduction. Journal of Phycology 41: 590-605.

Cecy, I.T. 1990. A Restinga do Pontal do Sul, município de Paranaguá, Pr. I-Levantamento ficológico (Euglenophyta) e físico-químico. Arquivos de Biologia e Tecnologia 33: 1-79.

Ciugulea, I., Nudelman, M.A., Brosnan, S. \& Triemer, R.E. 2008. Phylogeny of the Euglenoid loricate genera Trachelomonas and Strombomonas (Euglenophyta) inferred from nuclear SSU and LSU rDNA. Journal of Phycology 44: 406-418.

Conforti, V. 1993. Study of the Euglenophyta from Camaleão Lake (Manaus - Brazil) II. Strombomonas Defl. Revue Hydrobiological Tropical. 26: 187-197.

Conforti, V. \& Pérez, M.C. 2000. Euglenophyceae of Negro River, Uruguai, South America. Algological Studies 97: 59-78.

Conforti, V. \& Zalocar de Domitrovic, Y. 2003. Ultrastructural study of Strombomonas (Euglenophyta) from the Province of Chaco (Argentina). Boletin de la Sociedad Argentina de Botanica 38: 5-12.

Conforti, V.T.D., Walne, P.L. \& Dunlap, J.R. 1994. Comparative ultrastructure and elemental composition of envelopes of Trachelomonas and Strombomonas (Euglenophyta). Acta Protozoologica 33: 71-78.

Conrad, W. \& Van Meel, L. 1952. Matériaux pour une monographie de Trachelomonas Ehrenberg, C., 1834, Strombomonas Deflandre, G. 1930 et Euglena Ehrenberg, C., 1832, genres d'Euglénacées. Mémoires du Musée Royal d'Histoire Naturelle de Belgique 24: $1-176$.

Conti, J.B. \& Furlan, S.A. 2003. Geoecologia: o clima, os solos e a biota. In: J.L. Ross (org.). Geografia do Brasil. Editora da Universidade de São Paulo, São Paulo, pp. 67-207.

Deflandre, G. 1930. Strombomonas, nouveau genre d'eglenacées (Trachelomonas Ehr. pro parte). Archive für Protistenkund 69: 551-614.

Delazari-Barroso, A., Sant'Anna C.L. \& Senna, P.A.C. 2007. Phytoplankton from Duas Bocas Reservoir, Espírito Santo State, Brazil (except diatoms). Hoehnea 34: 211-229.

Domigues, C.D. \& Torgan. L.C. 2011. Fitoplâncton (exceto Chlorophyceae) de um lago artificial urbano no Sul do Brasil. Revista Brasileira de Botânica 34: 463-480.

Duangjan, K. \& Wolowski, K. 2013. New taxa of loricate euglenoids Strombomonas and Trachelomonas from Thailand. Polish Botanical Journal 58: 337-345.

Franceschini, I.M.F. 1992. Algues d'eau douce de Porto Alegre, Brésil (lês diatomophycées exclues). Bibliotheca Phycologica 92: 1-81.
Guiry, M.D. \& Guiry, G.M. 2016. Strombomonas. Worldwide electronic publication, National University of Ireland, Galway. Disponível em http://www.algaebase. org (acesso em 15-V-2015).

Hamm, A. 1969. Die Ermittlung der Gewässergüteklassen bei Flißgewäsern nach dem Gewässergütsystem und Gewässegutenomogramm. Münchner Beiträge zur Abwasser Fischrei und Flußbiologie 15: 46-48.

Hoek, C. van den, Mann, D.G. \& Jahns, H.M. 1995. Algae: An introduction to phycology. Cambrige University Press, Cambridge.

Huber-Pestalozzi, G. 1955. Euglenophyceen. In: G. HuberPestalozzi (ed.). Das phytoplankton des Susswässers: Systematik und Biologie. E. Schweizerbart'sche Verlangsbuchhandlug, Stuttgart 16: 1-605.

Jati, S. \& Train, S. 1994. Euglenaceae pigmentadas de ambientes lênticos da Ilha Porto Rico, município de Porto Rico, Paraná, Brasil. Iheringia, Série Botânica 45: 117-142.

Keppeler, E.C., Lopes, M.R.M. \& Lima, C.S. 2002. Ficoflorula do lago Amapá em Rio Branco-Acre, I: Euglenophyceae. Revista Brasileira de Biologia 59: 679-686.

Kim, J. I. \& Shin, W. 2008. Phylogeny of the Euglenales inferred from plastid LSU rDNA sequences. Journal of Phycology 44: 994-1000

Kim, J. I. \& Shin, W. \& Triemer, R. E. 2010. Multigene analyses of photosynthetic euglenoids and new family, Phacaceae (Euglenales). Journal of Phycology 46: 1278-1287.

Kim, J.I., Linton, E.W. \& Shin, W. 2015. Taxon-rich multigene phylogeny of the photosynthetic euglenoids (Euglenophyceae). Frontiers in Ecology and Evolution 2: $1-11$.

Linton, E. W., Karnkowska-Ishikawa, A., Kim, J. I., Shin, W., Bennett, M. \& Kwiatowski, J. 2010. Reconstructing euglenoid evolutionary relationships using three genes: nuclear SSU and LSU, and chloroplast 16S rDNA sequences and the description of Euglenaria gen. nov. (Euglenophyta). Protist 161: 603-619.

Marin, B., Palm, A., Klingberg, M. \& Melkonian, M. 2003. Phylogeny and taxonomic revision of plastidcontaining Euglenophytes based on SSU rDNA sequence comparisons and synapomorphic signatures in the SSU rDNA secondary structure. Protist 154: 99-145.

Matteucci, S.D. \& Colma, A. 1982. Metodologia para el estudio de la vegetacion. Secretaria General de la Organización de los Estados Americanos, Washington.

Menezes, M. 1992. Taxonomic studies on the Strombomonas verrucosa complex (Euglenophyta), Revista Brasileira de Biologia 52: 449-459.

Menezes, M. \& Fernandes, V.O. 1989. Euglenaceae (Euglenophyceae) pigmentadas do noroeste do Estado do Mato Grosso, Brasil: municípios de Barra dos Bugres, Cáceres, Juína e Porto Esperidião. Hoehnea 16: 35-55. 
Menezes, M., Fonseca, C.G. \& Nascimento, E.P. 1995. Algas de três ambientes de águas claras do município de Parintins, Estado do Amazonas, Brasil: Euglenophyceae e Dinophyceae, Hoehnea 22:1-15.

Nogueira, I.S. \& Leandro-Rodrigues, N.C. 1999. Algas planctônicas de um lago artificial do Jardim Botânico Chico Mendes, Goiânia, Goiás: florística e algumas considerações ecológicas. Revista Brasileira de Biologia 59: 377-395.

Philipose, M.T. 1988. Contribution to our knowledge of Indian algae 3. Euglenineae Part 3. The genera Trachelomonas Ehrenberg and Strombomonas Deflandre. Proceedings Indian Academy Sciences 98: 317-394.

Playfair, G.I. 1915. The genus Trachelomonas. Procedings of the Linnean Society of New South Wales 40: 1-41.

Popova, T.G. 1966. Evglenovyje vodoroski vyp. 1. Flora sporovych rastenijj Izd. Nauka, Moscou.

Pringshein, E.G. 1953. Observations on some species of Trachemononas grown in culture. New Phytologist 53: 93-113.

Rino, J.A. 1972. Contribuição para o conhecimento de algas de água doce de Moçambique. III. Revista de Ciências Biológicas 5: 121-164.

Rodrigues L. \& Bicudo D.C. 2001. Similarity among periphyton algal communities in a lentic-lotic gradient of the upper Paraná river floodplain, Brazil. Revista Brasileira de Botânica 24: 235-248.

Salazar, C. 2004. Euglenophyta pigmentadas asociadas a Hymenachne amplexicaulis en una sabana tropical inundable. Acta Botánica Venezuelica 27: 101-125.

Shi, Z., Wang, Q., Xie, S., Dai, J. \& Chen, L. 1999. Euglenophyta. (s.l.). Flora Algarum Sinicarum Aquae Dulcis, t. 6, Science Press, Beijing.

Silva, E.L. 2010. Bioma Pampa: Icmbio e a Área de Proteção Ambiental do Ibirapuitã. Disponível em http://sites.google.com/site/apadoibirapuita (acesso em 16-XI-2015).

Silva, C.B. 2011. Mapeamento das áreas de inundação do Rio Ibirapuitã em Alegrete, RS. Geografia Ensino \& Pesquisa 15: 73-92.
Starmach, K. 1983. Euglenophyta. In: K. Starmach (ed.). Flora Slodkowodna Polski, v. 3. Polska Academia Nauk, Warszawa.

Tell, G. \& Conforti, V.T.D. 1986. Euglenophyta pigmentadas de la Argentina. Biblioteca Phycologica 75: 1-301.

Triemer, R.E \& Zackrys, B. 2015. Photosynthetic Euglenoids. In: J.D. Wehr, R.G. Sheat, J.P. Kocioleck (eds.). Freshwater algae of North America. Ecology and classification. Academic Press. Cambridge. pp. 459-483.

Triemer, R.E., Linton, E., Shin, W., Nudelman, A., Monfils, A., Bennett, M. \& Brosnan, S. 2006. Phylogeny of the Euglenales based upon combined SSU and LSU rDNA sequence comparisons and description of Discoplastis gen. nov. (Euglenophyta). Journal of Phycology 42: 731-740.

Xavier, M.B. 1988. O gênero Strombomonas Deflandre do Parque Estadual das Fontes do Ipiranga, São Paulo, Brasil. In: J.G. Tundisi (ed.). Limnologia e Manejo de Represas. ACIESP/EESC/USP /CRHEA, São Paulo. pp. 473-505.

Xavier, M.B. 1994. Criptógamos do Parque Estadual das Fontes do Ipiranga, São Paulo, SP. Algas, 5: Euglenophyceae (Euglenaceae pigmentada). Hoehnea 21: 47-73.

Yacubson, S. 1980. The phytoplanckton of some freshwater bodies from Zulia State (Venezuela). Nova Hedwigia 33: 279-339.

Zaburlín N.M, Goñi O. \& Conforti V. 2004. Estúdio taxonômico de las Strombomonas (Euglenophyta) del Arroyo Yacarey, provincia de Corrientes, Argentina. Iheringia, Série Botânica 59: 117-130.

Wolowski, K. 1998. Taxonomic and environmental studies on euglenophytes of the Kraków-Czestochowa Upland (Southern Poland). Fragmenta Floristica et Geobotanica 6: 1-192.

Wolowski, K. \& Walne, P.L. 2007. Strombomonas and Trachelomonas species (Euglenophyta) from south-eastern USA, European Journal of Phycololgy 42: 409-431. 